\title{
Similarity Maximization of a Scaled Aeroelastic Flight Demonstrator via Multidisciplinary Optimization
}

\author{
Joan Mas Colomer*, Nathalie Bartoli ${ }^{\dagger}$, Thierry Lefebvre ${ }^{\dagger}$ and Sylvain Dubreuil ${ }^{\ddagger}$ \\ ONERA - The French Aerospace Lab, Toulouse, France \\ Joaquim R. R. A. Martins ${ }^{\S}$ \\ University of Michigan, Ann Arbor, Michigan 48109-2102 \\ Emmanuel Bénard \\ Université de Toulouse, ISAE SUPAERO, Toulouse, France \\ Joseph Morlier" \\ Institut Clément Ader (ICA), Université de Toulouse, \\ ISAE SUPAERO-CNRS-INSA-Mines Albi-UPS, Toulouse, France
}

\begin{abstract}
The developments presented in this paper take place in the context of a broader series of works carried out at ONERA and ISAE on multidisciplinary design optimization applied to a scaled flight demonstrator. The aim of this work is to develop an optimization process capable of sizing a scaled flight demonstrator in order to reproduce several behaviors encountered on its corresponding full size aircraft. Unlike the classical optimization problems found in aeronautics, whose objective functions are performance-related (e.g. mass and drag minimization), we aim to maximize the similarity between the scaled model and the full size aircraft. In the aforementioned context, the first part of this paper corresponds to the static aeroelastic similarity problem. However, the approach described herein is general enough to treat other optimization problems, including performance-related ones. The second part of this work deals with the dynamic aspects of the aeroelastic similarity. A benchmark case is presented where the structural properties of a given geometry are optimized in order to match the reference modal parameters (i.e., mode shapes and frequencies) of the GARTEUR SM-AG19 model.
\end{abstract}

\section{Nomenclature}

()$^{*} \quad$ Functions or variables at their optimal value

()$^{0} \quad$ Variables at their initial value

()$^{T} \quad$ Transpose of a matrix or vector

()$^{t} \quad$ Current copy of a state variable

$\left[\omega_{r e f}\right]$ Vector of reference eigenfrequencies

$\left[\Phi_{r e f}\right]$ Matrix of reference eigenvectors

$[m] \quad$ Concentrated masses vector

$[t] \quad$ Skin thickness vector

$\alpha \quad$ Angle of attack

*PhD Student, System Design and Performance Evaluation Department, AIAA Student Member.

${ }^{\dagger}$ Research Engineer, System Design and Performance Evaluation Department, AIAA Member.

¥Postdoctoral Researcher, System Design and Performance Evaluation Department.

§Professor, Department of Aerospace Engineering, AIAA Associate Fellow.

ฯAssociate Professor, Department of Aerospace Vehicles Design and Control, AIAA Member.

" Professor, Structural Mechanics Department, AIAA Member. 


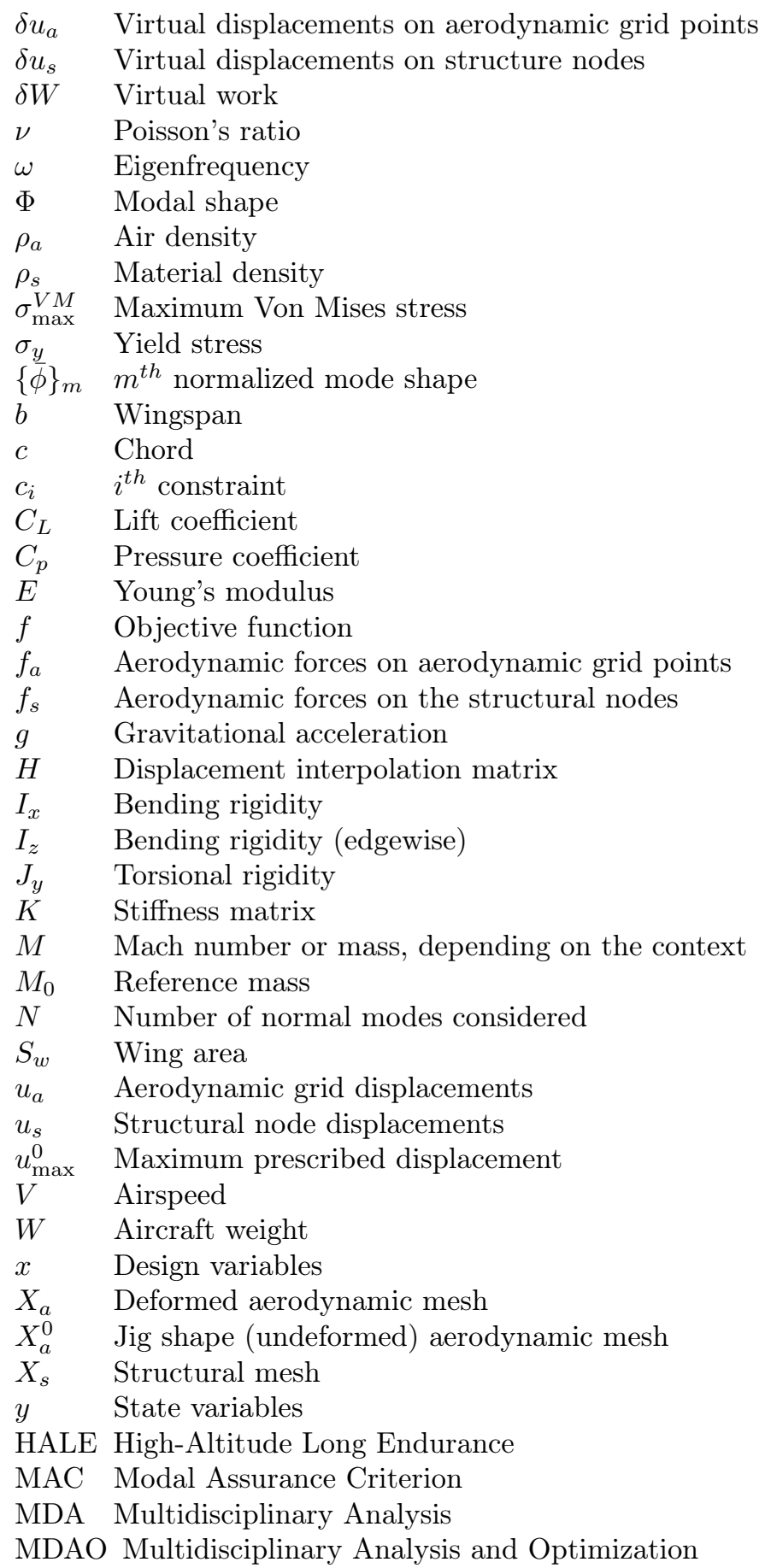

\section{Introduction}

7 HE problem of data transposition between a scaled model and its corresponding full size aircraft can 1 be usually treated by similitude analysis when the complete geometry (including the internal structure architecture) of the model is a scaled version of the full size aircraft. However, due to technological limitations and simplifications of the model construction, it is not usually possible to achieve a scaled version of the complete structure of the aircraft. It is for that reason that the design parameters defining the model structure cannot be obtained directly by the use of scaling factors derived from similitude analysis. In that situation, the use of optimization techniques emerges as a solution in order to minimize the difference between the response of the model and the scaled response of the complete aircraft. For the present case, 
these responses are wing deflections for a given flight condition, structural normal modes and frequencies, and flutter speeds.

One of the reasons to implement a static aeroelastic optimization process for the purpose of similarity studies is the fact that the flight conditions (e.g. Mach number) generally differ between the scaled model and the full size aircraft. As an example, Richards et al. ${ }^{1}$ achieve static deflection similarity through the stiffness matching, since they obtain aerodynamic similarity by preserving the outer mold line of the original geometry. This holds for the particular case where there exists flow similarity (e.g. Mach number) between the two cases. However, when flow similarity cannot be achieved, due to a restricted flight envelope, for example, , the lift distribution changes. Therefore, a coupled aeroelastic analysis is required in order to establish the deflection similarity. The recent work by Ricciardi et al. ${ }^{2}$ is an example of the current developments on the subject of aeroelastic scaled models.

Concerning the case of dynamic similarity (i.e. scaled flutter speeds, modes and frequencies), Ricciardi et al. ${ }^{3}$ establish the conditions required for the dynamic aeroelastic similitude. They show that, under certain assumptions such as flow similarity guaranteed through a scaled aerodynamic shape and the same Mach number, the dynamic aeroelastic similitude problem reduces to the minimization of the difference between the structural normal modes and frequencies of the model, and the scaled modal parameters of the full size aircraft.

The difference between the response of the scaled model and the complete aircraft constitutes the objective function that has to be minimized for this optimization problem. The performance capabilities of the model (such as a minimum range or endurance) or a certain scaled mass constitute the constraints of the problem.

Figure 1 shows the $\mathrm{XDSM}^{\mathrm{a} 4}$ diagram representing the optimization problem proposed to address the issue of aeroelastic similarity of flight demonstrators. On an XDSM diagram, each rectangular box represents an analysis (e.g. a function or a computational code), whose input variables are placed on the vertical that passes through the analysis box and whose outputs are on the horizontal line. Thick gray lines represent data dependencies, whereas thin black lines represent process connections. Components are numbered to establish the order in which they are executed.

The steps that define the optimization process are the following:

0 . Initiate the optimization process.

1. From the aerodynamic and structural design variables, compute flutter modes and speeds, static aeroelastic shape, drag, lift, stresses, and mass.

2. From the drag value and mission design variables, compute the range and endurance. At the same time, compute the objective function, which depends on the flutter modes and speeds and on the static deformed shape.

3. Compute the constraints related to the mission performance from the range and endurance. From the results of the static analysis, compute the constraints related to maximum stresses and displacements. From the lift force, compute the lift constraint.

4. Based on the objective and constraint values, decide the design variables values for the next optimization iteration.

Steps 1 to 4 are repeated until the convergence of the optimization is achieved. The process shown in Figure 1 is not implemented in this work, it is only intended to give the reader an idea of the complete similarity process applied to flight demonstrators.

\footnotetext{
aThe XDSM (eXtended Design Structure Matrix) diagram is a tool developed by Lambe and Martins ${ }^{4}$ that aims to represent MDO processes.
} 


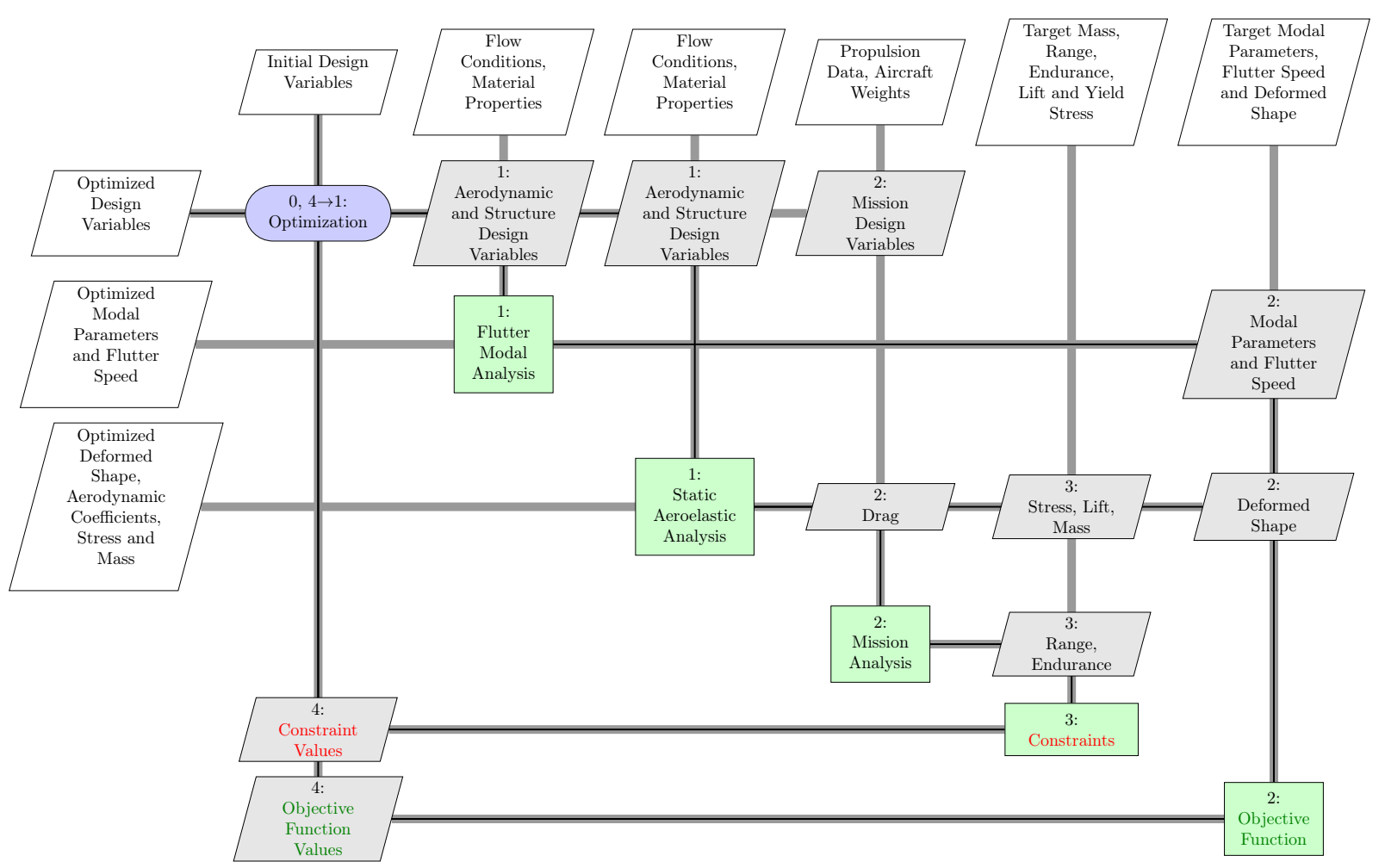

Figure 1. $\mathrm{XDSM}^{4}$ diagram of the multidisciplinary analysis and optimization process for the similarity maximization of a scaled flight demonstrator.

In this work, we focus on the aeroelastic optimization part of the problem. The first part of this paper (sections II and III) deals with static aeroelasticity. The second part of the paper focuses on the dynamic aeroelastic similarity and its associated optimization process. All the multidisciplinary analysis and optimization processes presented in this work are implemented using the OpenMDAO ${ }^{5}$ framework.

First, the aerodynamic and structural codes used for this coupling are presented, as well as the displacement and force transfer scheme used to exchange data between them. This static aeroelastic coupling process, which will be used on further optimizations in this work, is checked against a test case proposed by Patil and Hodges ${ }^{6}$ which corresponds to a HALE (High-Altitude Long Endurance) wing. This test case is not specific to the subject of aeroelastic similarity, but constitutes a benchmark used by several authors in the literature.

Next, a static aeroelastic optimization process that makes use of the static coupling mentioned above is presented. An aeroelastic optimization problem is defined in order to test the process. This problem consists in the minimization of the structural mass of the previously presented wing with constraints on lift, stress and maximum displacement. However, the original wing structure (represented by a beam) is replaced by a wingbox configuration, modeled by shell elements. The outer mold line of the aerodynamic surface remains the same.

The dynamic similarity part of this work presents the conditions that must be satisfied to achieve it as described by Ricciardi et al. ${ }^{3}$ As this similarity problem is reduced to a structural modal optimization, we define the new objective function and constraints of this optimization problem. This type of objective function is based on the Modal Assurance Criterion (MAC) mode-tracking in order to avoid discontinuities on the objective and constraint functions as explained by Kim and Kim. ${ }^{7}$ Finally, we apply this modal optimization process to the GARTEUR SM-AG19 ${ }^{8}$ model, were several plate thicknesses and point mass values are optimized in order to reproduce the vibration modes and frequencies of a reference configuration. 


\section{Static Aeroelastic Coupling}

The multidisciplinary analysis (MDA) is based on the static aeroelastic coupling between a linear structural model and an inviscid potential flow model. An open-sourced version of NASA's NASTRAN95 ${ }^{9}$ is used as the linear finite element solver for the structural problem. For the aerodynamics, we selected an open-sourced version of NASA's and Boeing's PANAIR/A502 panel code. ${ }^{10}$

Given a wing structural configuration, outer aerodynamic shape, and angle of attack, the wing will reach a certain static deformed state. This static aeroelastic equilibrium corresponds to the state where the aerodynamic forces acting on the deformed wing match those necessary to produce that particular deflection. This aeroelastic coupling is implemented by using the OpenMDAO framework. ${ }^{5}$

The static aeroelastic equilibrium is achieved using fixed-point iteration, implemented through a nonlinear Gauss-Seidel iteration under OpenMDAO. In that case, the stopping criterion is a variation of the tip deflection between iterations of less than $2 \%$. This coupling constitutes the multidisciplinary analysis (MDA) loop.

Since the structural and aerodynamic grids are, in the general case, not coincident, coupling data must be interpolated between them in order to transfer the aerodynamic loads to the structural model, and deform the aerodynamic grid according to the structural displacements.

In order to accomplish this data exchange, a fluid-structure interpolation and mesh motion scheme based on the use of radial basis functions (RBF) has been used. This method is presented in the work by Rendall and Allen. ${ }^{11}$ This displacement and load transfer technique is conservative in terms of total load and moment, as shown by Jakobsson and Amoignon. ${ }^{12}$ One of the advantages of this type of interpolations is that no mesh connectivity is required between the two disciplines. This is particularly suitable for the cases where the aerodynamic and structural models do not represent the same geometries. Usually, the aerodynamic grid is based on the outer mold line, even though there may be cases where it is based on the mean camber surface only (e.g., the vortex lattice methods). However, the structural model is often only representative of the wingbox or is idealized as a beam model. In the following subsections, we describe the two disciplines involved in this coupling, the displacement and force transfer method used, the formulation of the problem, and its corresponding results on a benchmark case.

\section{A. Aerodynamics}

The aerodynamic loads are computed by means of a potential flow panel code (PANAIR/A502) ${ }^{10}$ which, given an aerodynamic grid and angle of attack determines the pressure coefficient $\left(C_{p}\right)$ at the control points of the panels. However, in order to apply the RBF based load and displacement transfer scheme, aerodynamic loads have to be determined on the aerodynamic grid points. This is achieved by numerically integrating the $C_{p}$ distribution over the aerodynamic panels and then evenly distributing the total panel force among the four panel vertices, which belong to the aerodynamic grid. To account for the aeroelastic effects, the aerodynamic grid must be deformed at each MDA iteration. A symmetric flow is assumed throughout this work.

\section{B. Structures}

A linear finite element model is used in order to compute the displacements of the wing. The equation that must be solved for the finite element analysis is

$$
K u_{s}=f_{s},
$$

which is linear with respect to the structural displacements, as the stiffness matrix $K$ depends only on the undeformed geometry (jig shape) of the wing and its material properties.

In order to solve this linear system, Nastran uses an LU decomposition of the stiffness matrix $K$. Since the only term that changes at each MDA iteration is the right hand side of Eq. (1) (i.e., the current aerodynamic loads), the same LU decomposition may be used for all the iterations. This can be achieved in Nastran by means of a DMAP alter sequence. ${ }^{9}$ Here, the LU decomposition of $K$ is stored at the beginning of the loop and reused at every MDA iteration. 


\section{Displacement and Load Transfer}

The coupling variables of the MDA are: structural node displacements $u_{s}$, aerodynamic grid displacements $u_{a}$, aerodynamic forces on aerodynamic grid points $f_{a}$, and aerodynamic forces on the structural nodes $f_{s}$.

Since the structural and aerodynamic meshes are not coincident, the structural displacements, $u_{s}$, must be interpolated onto the aerodynamic grid points. The displacement interpolation scheme is based on the work by Rendall and Allen. ${ }^{11}$ In that method, each component of the displacement vector $\mathbf{u}$ is interpolated as follows (Eq. (2) is written for the $x$ component, but the same holds for $\mathrm{y}$ and $\mathrm{z}$ ):

$$
u_{x}=\sum_{i=1}^{N_{s}} \alpha_{i}^{x} \phi\left(\left\|\mathbf{x}-\mathbf{x}_{\mathbf{i}}\right\|\right)+\gamma_{0}^{x}+\gamma_{x}^{x} x+\gamma_{y}^{x} y+\gamma_{z}^{x} z,
$$

where $\phi(r)$ is the form of function adopted. In that case, we choose $\phi(r)=r^{2} \ln r$, known as the Thin Plate Spline function (TPS). According to Lombardi et al., ${ }^{13}$ who performed a comparison between several available functions, the use of TPS functions is the best and safest option in terms of accuracy of the interpolation. The terms $\alpha_{i}^{x}$ are the coefficients of the radial basis functions. Each structural node is the center of an $\operatorname{RBF}\left(\mathbf{x}_{\mathbf{i}}\right)$ and the $\gamma$ terms are the coefficients of the linear polynomial part. By imposing the interpolating condition on these coefficients (the interpolation function evaluated at the structural nodes must be equal to their known displacements) and by evaluating this same function on the aerodynamic grid points, the transformation matrix between the displacements of the structural and aerodynamic points can be expressed as

$$
u_{a}=H u_{s}
$$

where $H$ is a matrix which depends only on the coordinates of the structural and aerodynamic grid points and the type of RBF chosen. Figure 2 shows the deformed aerodynamic grid points (in blue) when the interpolation matrix on Eq. (3) is applied to the structural displacements. The structural displacements, $u_{s}$, can be visualized as the difference between the deformed state (red points) and the structure at rest (in green).

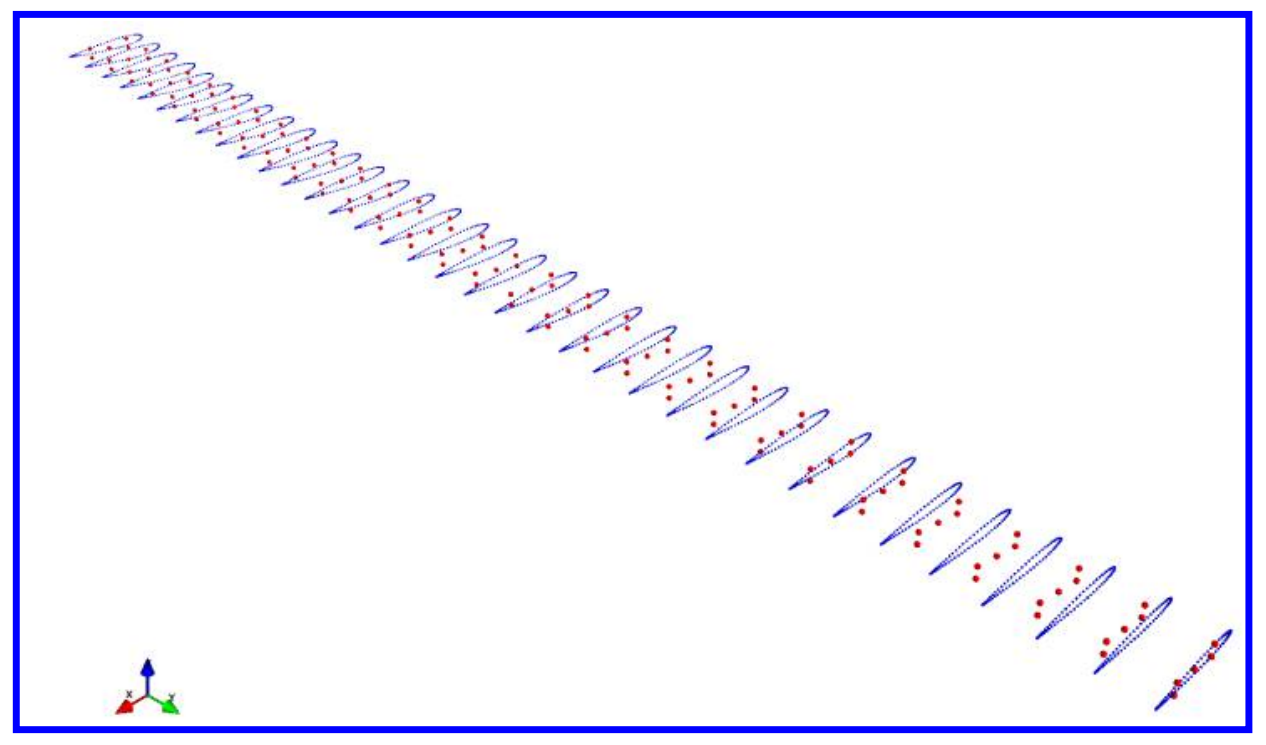

Figure 2. Example of aerodynamic grid (blue) and structure nodes (red).

As detailed by Rendall and Allen, ${ }^{11}$ and by virtue of the principle of virtual work to ensure the conservation of energy, we can determine the transformation matrix between the aerodynamic forces on the aerodynamic $\left(f_{a}\right)$ and structural $\left(f_{s}\right)$ points. The virtual work can be written as

$$
\delta W=\delta u_{s}^{T} \cdot f_{s}=\delta u_{a}^{T} \cdot f_{a},
$$


where $\delta u_{s}$ and $\delta u_{a}$ are the virtual displacements of the structural and aerodynamic grids respectively. Through the displacement interpolation matrix $H$, we can express the virtual displacements of the aerodynamic grid

$$
\delta u_{a}=H \delta u_{s}
$$

as a function of $\delta u_{s}$. By substituting equation (5) into equation (4) we get that

$$
f_{s}=H^{T} f_{a} .
$$

In the case where gradient-based optimization techniques are used for optimization problems that use the aerostructural coupling presented herein, it can be useful to compute the partial derivatives of the coordinates of the deformed aerodynamic mesh $X_{a}$ with respect to the structural displacements $u_{s}$, as well as the partial derivatives of the aerodynamic forces on the structural nodes $f_{s}$ with respect to the forces on the aerodynamic grid points $f_{a}$. The deformed aerodynamic mesh is obtained by adding the interpolated displacements (given by equation (3)) to the jig shape aerodynamic mesh:

$$
X_{a}=X_{a}^{0}+u_{a}=X_{o}+H u_{s}
$$

Therefore, by considering equations (6) and (7), these partial derivatives can be expressed in terms of the interpolation matrix $H$ as

$$
\begin{aligned}
& \frac{\partial X_{a}}{\partial u_{s}}=H \\
& \frac{\partial f_{s}}{\partial f_{a}}=H^{T}
\end{aligned}
$$

\section{Problem definition}

In order to test the aeroelastic coupling, we use the test case presented by Patil and Hodges ${ }^{6}$ as a benchmark. In this test case, a HALE wing, whose properties and flight conditions are detailed in Table 1, is studied and the obtained results are compared.

\begin{tabular}{lcccc} 
Model Data & \multicolumn{3}{l}{ Flight Conditions } \\
\cline { 1 - 2 } \cline { 5 - 5 } Half-span, $b / 2$ & $16 \mathrm{~m}$ & & Altitude & $20 \mathrm{~km}$ \\
Chord, $c$ & $1 \mathrm{~m}$ & & Density of air & $0.0889 \mathrm{~kg} / \mathrm{m}^{3}$ \\
Spanwise elastic axis & $50 \%$ chord & & Mach number & $0.0847(25 \mathrm{~m} / \mathrm{s})$ \\
Center of gravity & $50 \%$ chord & & Angle of attack & $2^{\circ}$ \\
Bending rigidity, $I_{x}$ & $2 \times 10^{4} \mathrm{Nm}^{2}$ & & \\
Torsional rigidity, $J_{y}$ & $1 \times 10^{4} \mathrm{Nm}^{2}$ & & \\
Bending rigidity (edgewise),$I_{z}$ & $5 \times 10^{6} \mathrm{Nm}^{2}$ & & \\
Airfoil & NACA 0012 & &
\end{tabular}

Table 1. Model data and flight conditions for the test wing (SI units)

For this case, 30 beam elements, placed along the mid-chord, have been used to represent the wing structure with the given structural properties. For every beam element node (excluding the root section one), 4 additional nodes have been placed, linked through rigid bars to the beam nodes, in order to have a set of non-coplanar points. This is necessary because otherwise the interpolation matrix would be singular, as explained by Lombardi et al. ${ }^{13}$ This configuration is shown on Figure 3. 


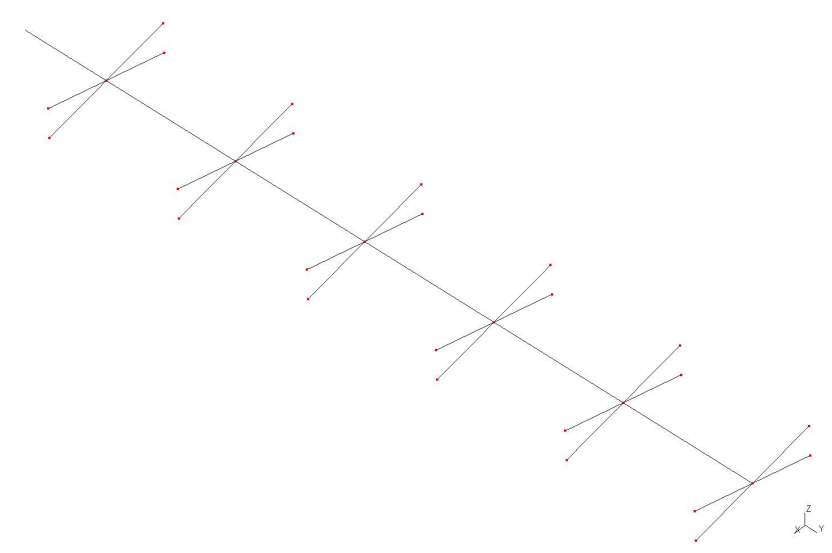

Figure 3. Detail of the rigid bars attached to the nodes of the beam.

Figure 4 shows the $\mathrm{XDSM}^{4}$ diagram corresponding to the presented aeroelastic coupling process.

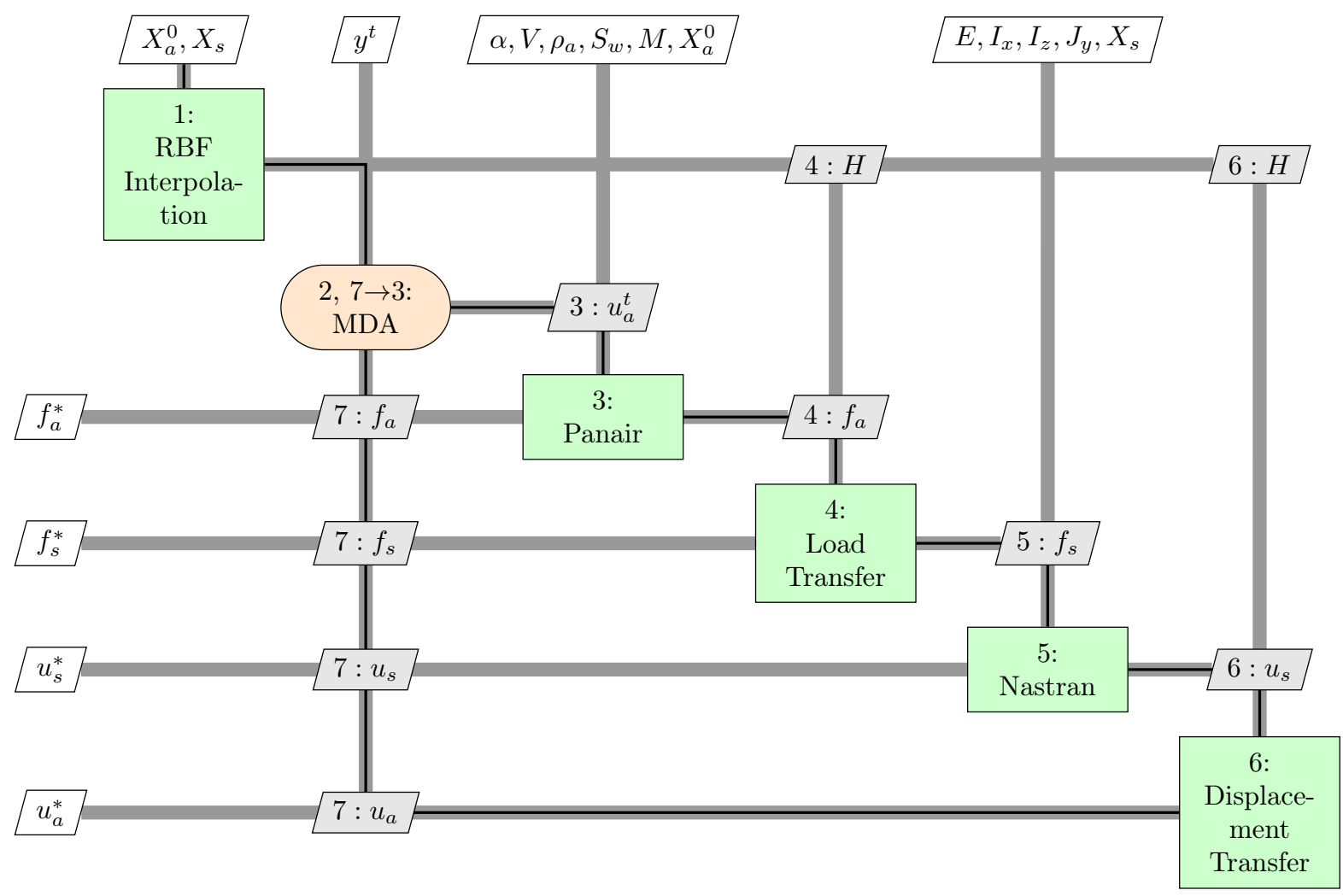

Figure 4. $\mathrm{XDSM}^{4}$ representation of the implemented aeroelastic coupling.

Algorithm 1 describes the sequence of operations performed to carry out the MDA shown on figure 4 . 


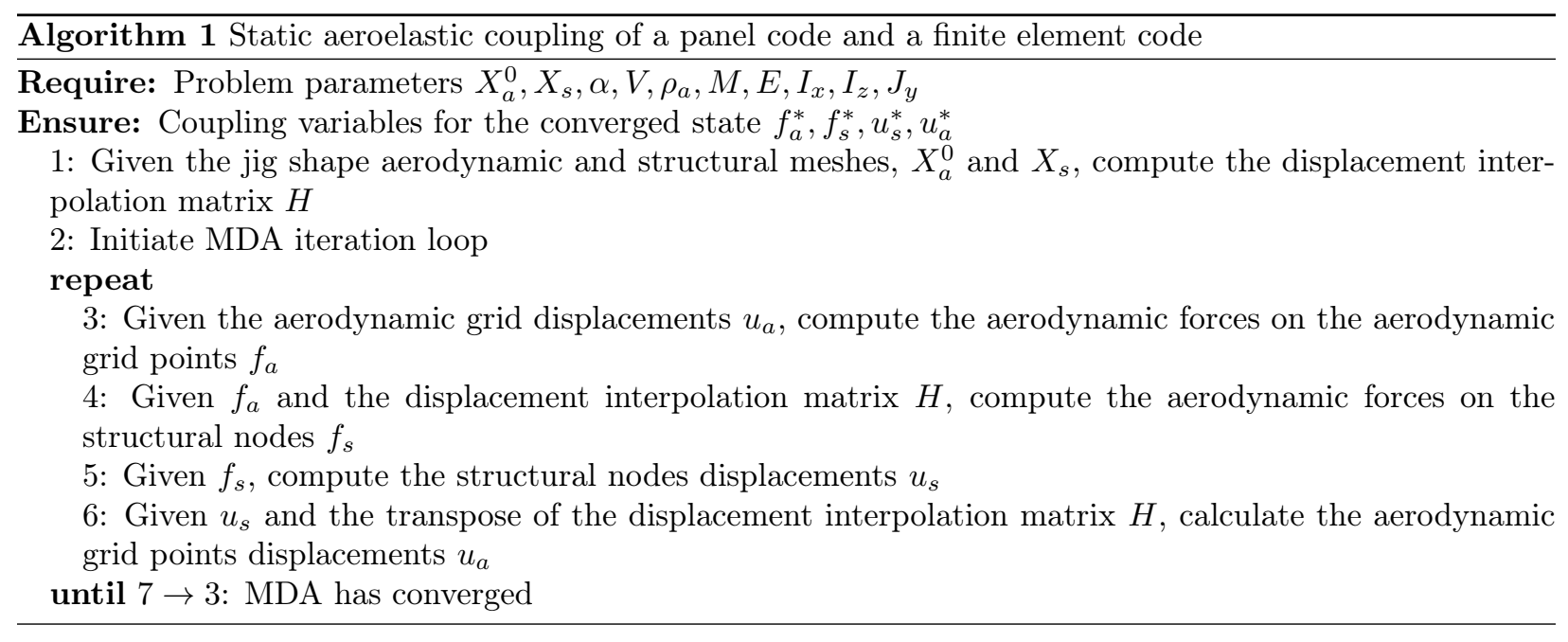

\section{E. Results}

After the multidisciplinary analysis has converged for the specified problem parameters, we can compare the obtained results to the ones found in the literature. ${ }^{6,14}$

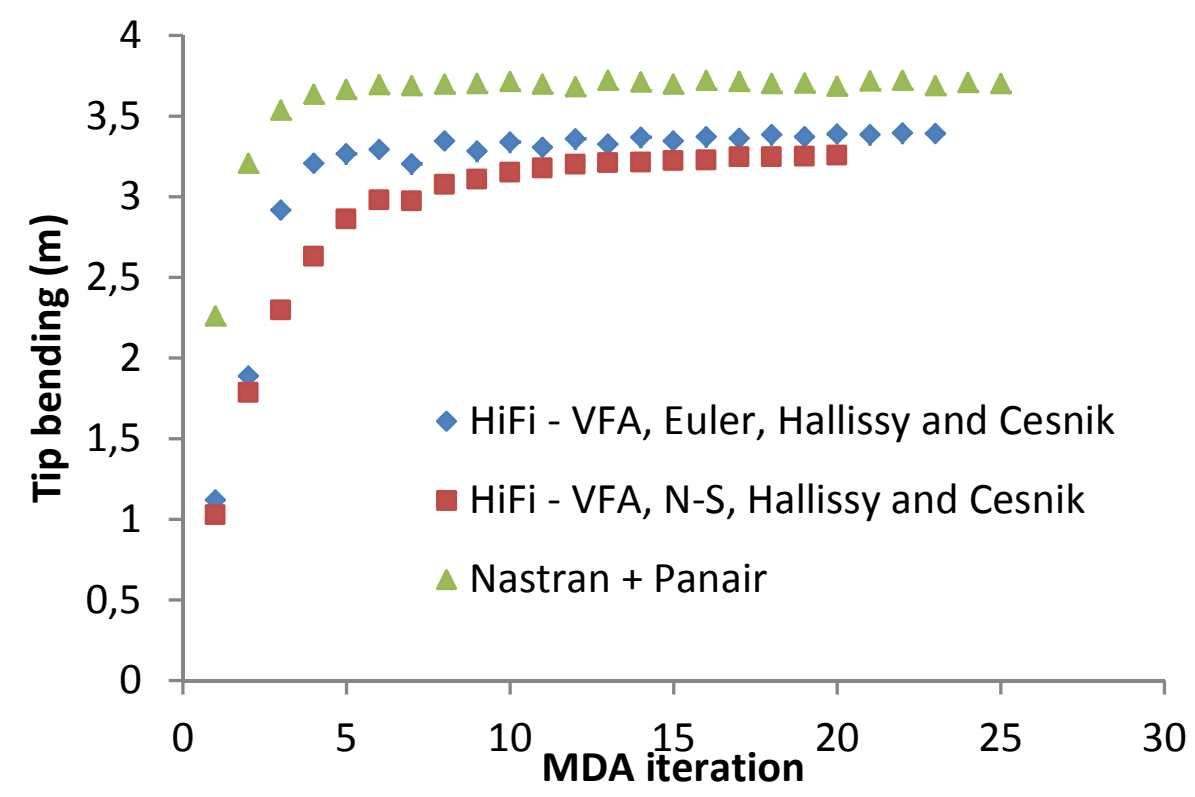

Figure 5. Tip bending deflection convergence compared to the ones by Hallissy and Cesnik. ${ }^{14}$

On Figure 5, the tip deflection is represented as a function of the number of iterations. Since no convergence data was available from Patil and Hodges, ${ }^{6}$ we use the results of Hallissy and Cesnik. ${ }^{14}$ The steady deflection is lower for the Euler and Navier-Stokes cases as the computed aerodynamic loads are lower. 


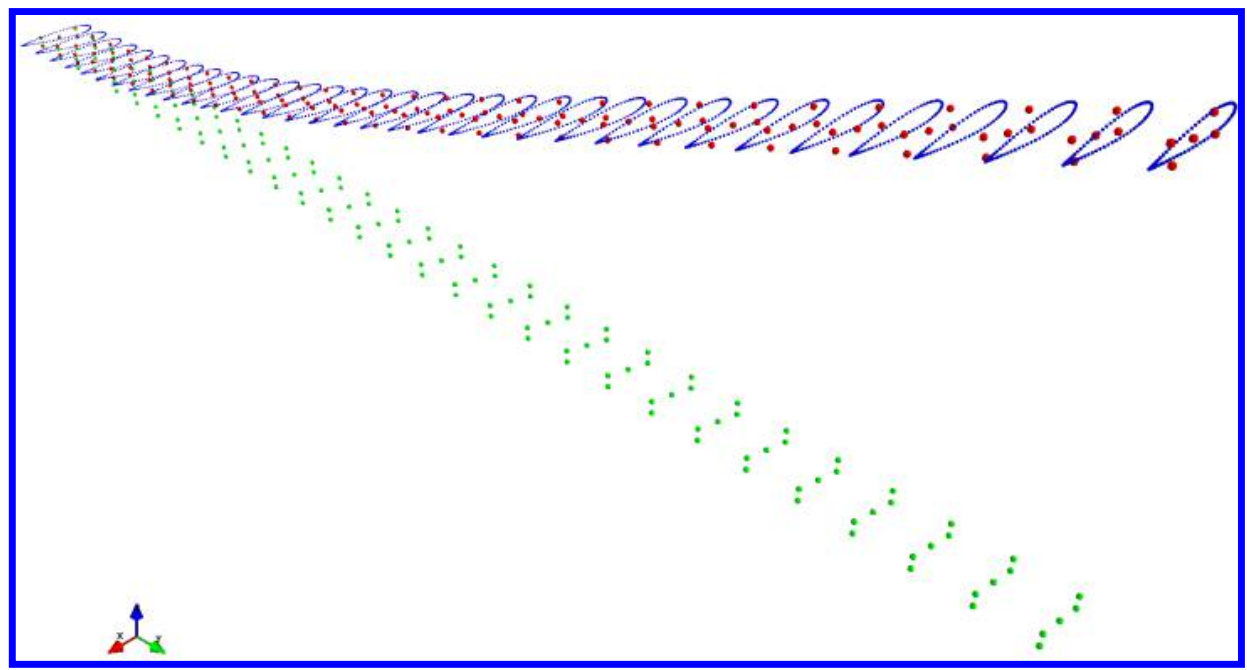

Figure 6. Converged deformed shape: Aerodynamic points (blue), structure nodes (red).

Figure 6 shows the deformed state of the structural nodes (in red) as well as the deformed aerodynamic grid points (in blue).
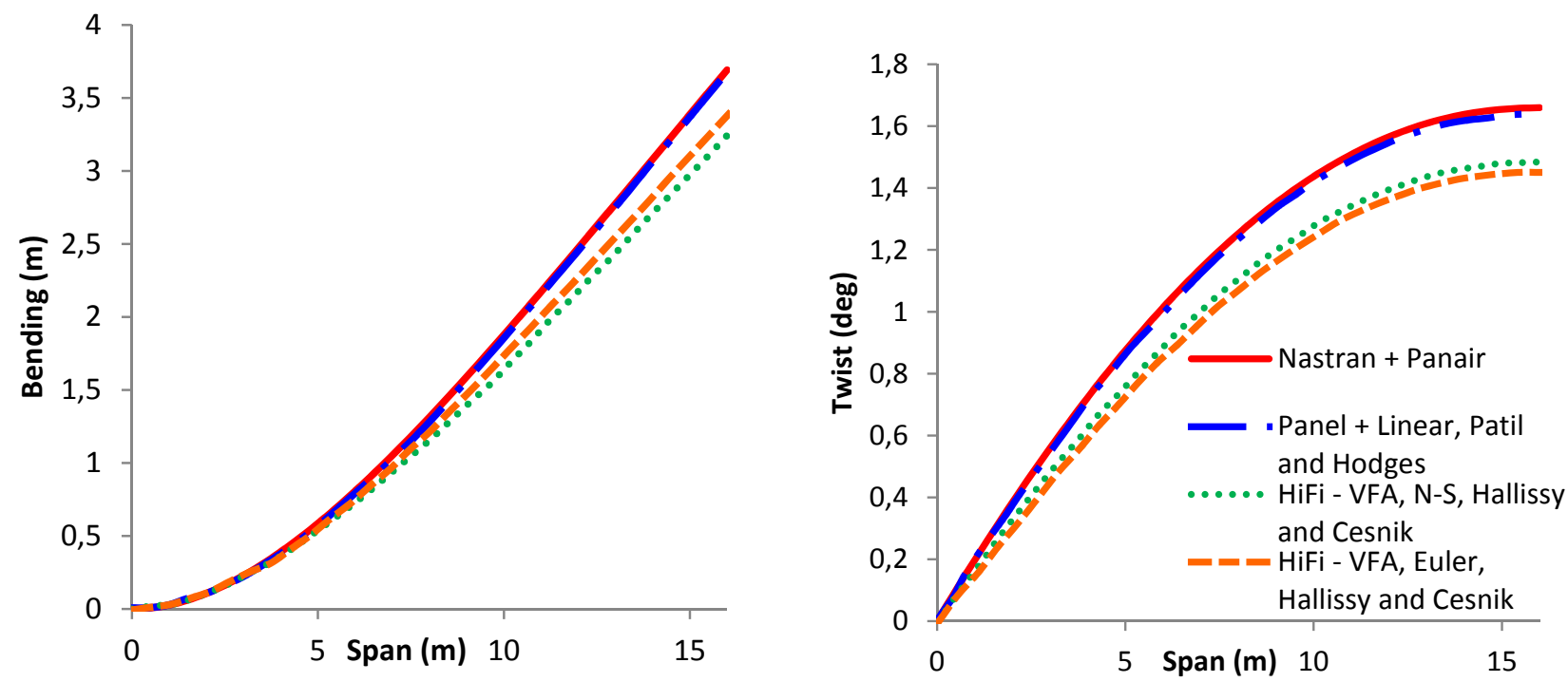

Figure 7. Spanwise deflection (left) and twist (right). Comparison between the presented approach (Nastran+Panair), and results from references. ${ }^{6,14}$

Figure 7 displays the bending deflection of the wing as well as the section twist along the wingspan, compared to the results found in the literature. ${ }^{6,14}$ We obtain a relative error of $0.37 \%$ in the wingtip deflection between the implemented coupling of NASTRAN95 and PANAIR/A502 and the results obtained by Patil and Hodges ${ }^{6}$ with a panel method and linear FEM model. Figure 8 depicts the $C_{p}$ distribution of the converged state on the deformed wing, as well as the transferred aerodynamic loads to the beam model.

Once the implementation of this static aeroelastic coupling has been validated, we will use it in the following section for an aeroelastic optimization problem of a HALE wing. 

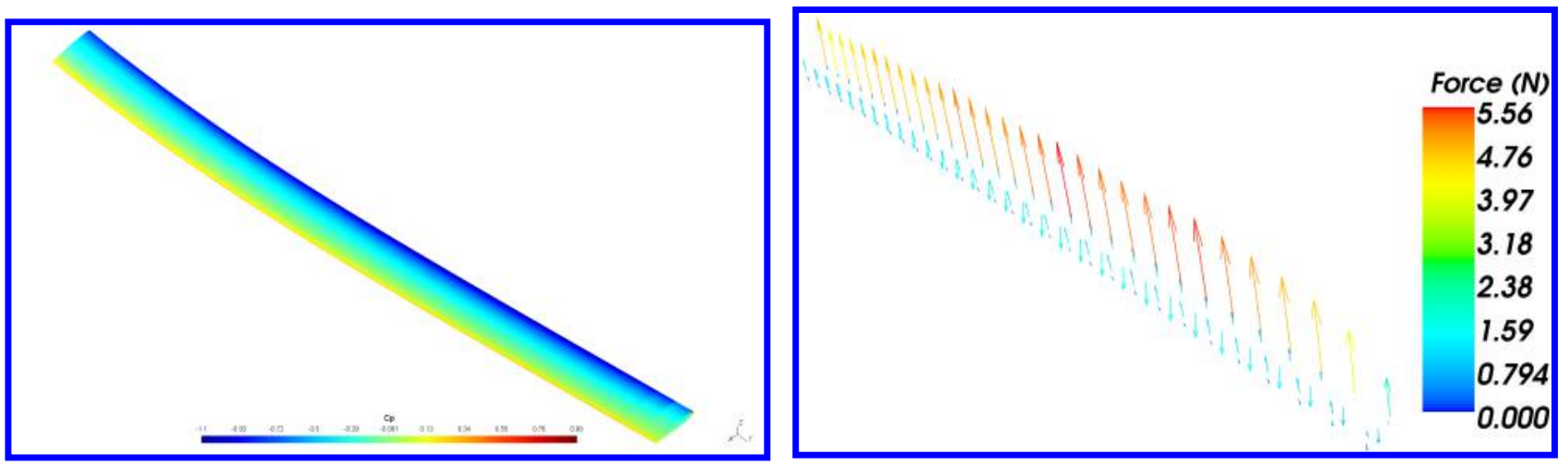

Figure 8. Computed aerodynamic (left) and transferred structural loads (right).

\section{Aeroelastic Optimization}

\section{A. Problem Formulation}

A first test case is used with the sole aim of validating the optimization process based on the aeroelastic analysis presented above. This test case uses the same outer mold line of the HALE wing presented previously. However, the beam model is substituted by a skins, spars and ribs configuration, represented by shell elements. The flow conditions and required lift represent the flight of a typical HALE aircraft. The objective is to minimize the structural mass of the wing while satisfying constraints on the maximum displacements, the maximum von Mises stress, and the required lift. For the constraints on the displacements, the maximum wing deflection is set to $30 \%$ of the half wingspan. For that case, the structural model is built from shell elements representing the upper and lower skins, the front and aft spars, and 4 ribs. Each one of these structural components is characterized by a thickness. This makes a total of 8 thickness values, which are considered as design variables of the problem. Figure 9 depicts the different thickness regions of the wing. The angle of attack is also an independent design variable of the problem, in order to achieve the required lift. Table 2 shows the objective function, the 9 design variables and the 3 constraints of the problem.

\begin{tabular}{lcll} 
Objective Function & & Dimension & Bounds \\
\hline Mass & $\min (M)$ & $\mathbb{R}$ & \\
Design Variables & & & \\
\hline Angle of attack & $\alpha$ & $\mathbb{R}$ & {$[0,10]^{\circ}$} \\
Skin thickness vector & {$[t]$} & $\mathbb{R}^{8}$ & {$[0.001,0.01] \mathrm{m}$} \\
Constraints & & & \\
\hline Yield stress & $\sigma_{\max }^{V M}-\sigma_{y} \leq 0$ & $\mathbb{R}$ & \\
Maximum displacement & $\max \left(u_{s}\right)-0.3 \frac{b}{2} \leq 0$ & $\mathbb{R}$ & \\
Lift & $C_{L}-\frac{W g}{\frac{1}{2} \rho_{a} V^{2} S_{w}}=0$ & $\mathbb{R}$ &
\end{tabular}

Table 2. Static aerostructural optimization problem.

Table 3 lists values of the optimization problem input parameters. These values represent the weight, material properties, dimensions and flight conditions of a typical HALE aircraft. The considered material is a typical aluminum alloy. 


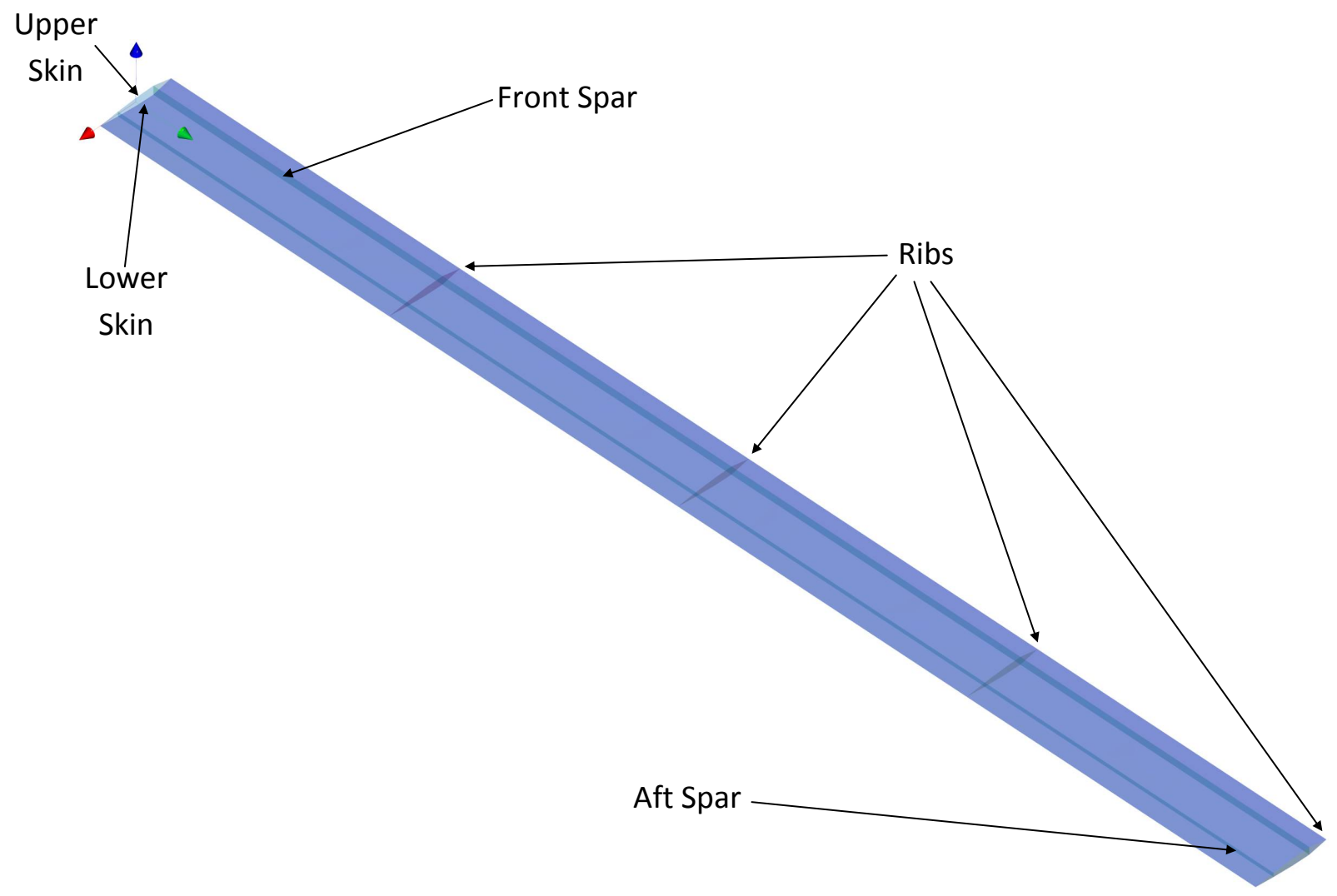

Figure 9. Thickness groups of the wing.

\begin{tabular}{lr} 
Quantity & Value \\
\hline Mach Number $M$ & 0.3093 \\
Wing area $S_{w}$ & $32 \mathrm{~m}^{2}$ \\
Wingspan $b$ & $32 \mathrm{~m}$ \\
Chord $c$ & $1 \mathrm{~m}$ \\
Air density $\rho_{a}$ & $0.653 \mathrm{~kg} / \mathrm{m}^{3}$ \\
Airspeed $V$ & $97.78 \mathrm{~m} / \mathrm{s}$ \\
Weight $W$ & $5000 \mathrm{~kg}$ \\
Young's modulus $E$ & $71.7 \mathrm{GPa}$ \\
Poisson's ratio $\nu$ & 0.33 \\
Material density $\rho_{s}$ & $2810 \mathrm{~kg} / \mathrm{m}^{3}$ \\
Yield stress $\sigma_{y}$ & $503.3 \mathrm{MPa}$
\end{tabular}

Table 3. Input parameters for the static aeroelastic optimization test case.

Figure 10 shows the $\mathrm{XDSM}^{4}$ diagram for the aeroelastic optimization process. The inner part of this diagram corresponds to the aeroelastic coupling loop in Figure 4. In comparison to the latter, we can see that the optimizer constitutes the outer loop, and that the evaluation of the objective function and constraints (step 9) have been added.

Algorithm 2 describes the sequence of operations for the MDA and optimization shown on figure 10. This corresponds to the multidisciplinary feasible MDO architecture. ${ }^{15}$ 


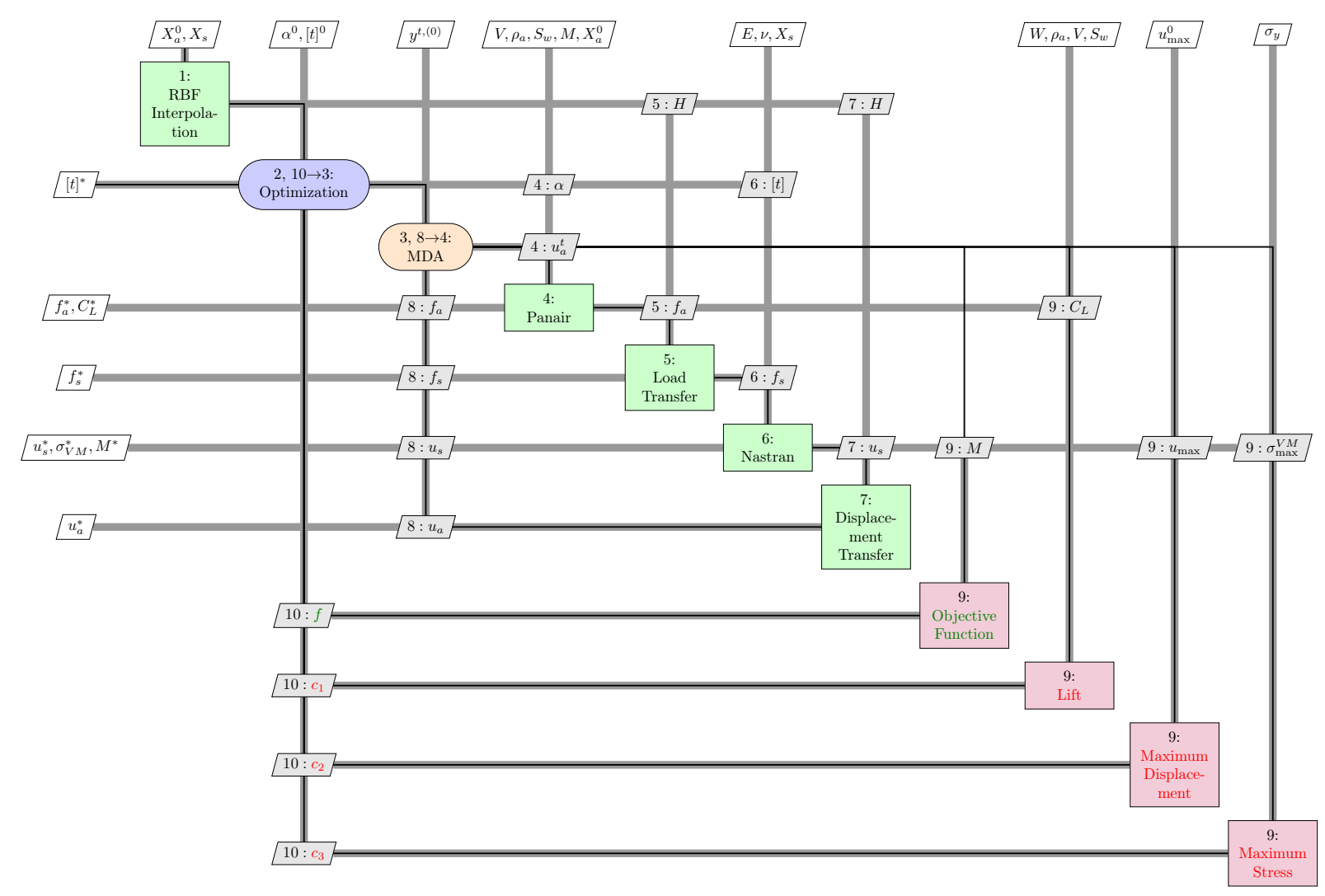

Figure 10. $\mathrm{XDSM}^{4}$ representation of the implemented aeroelastic optimization.

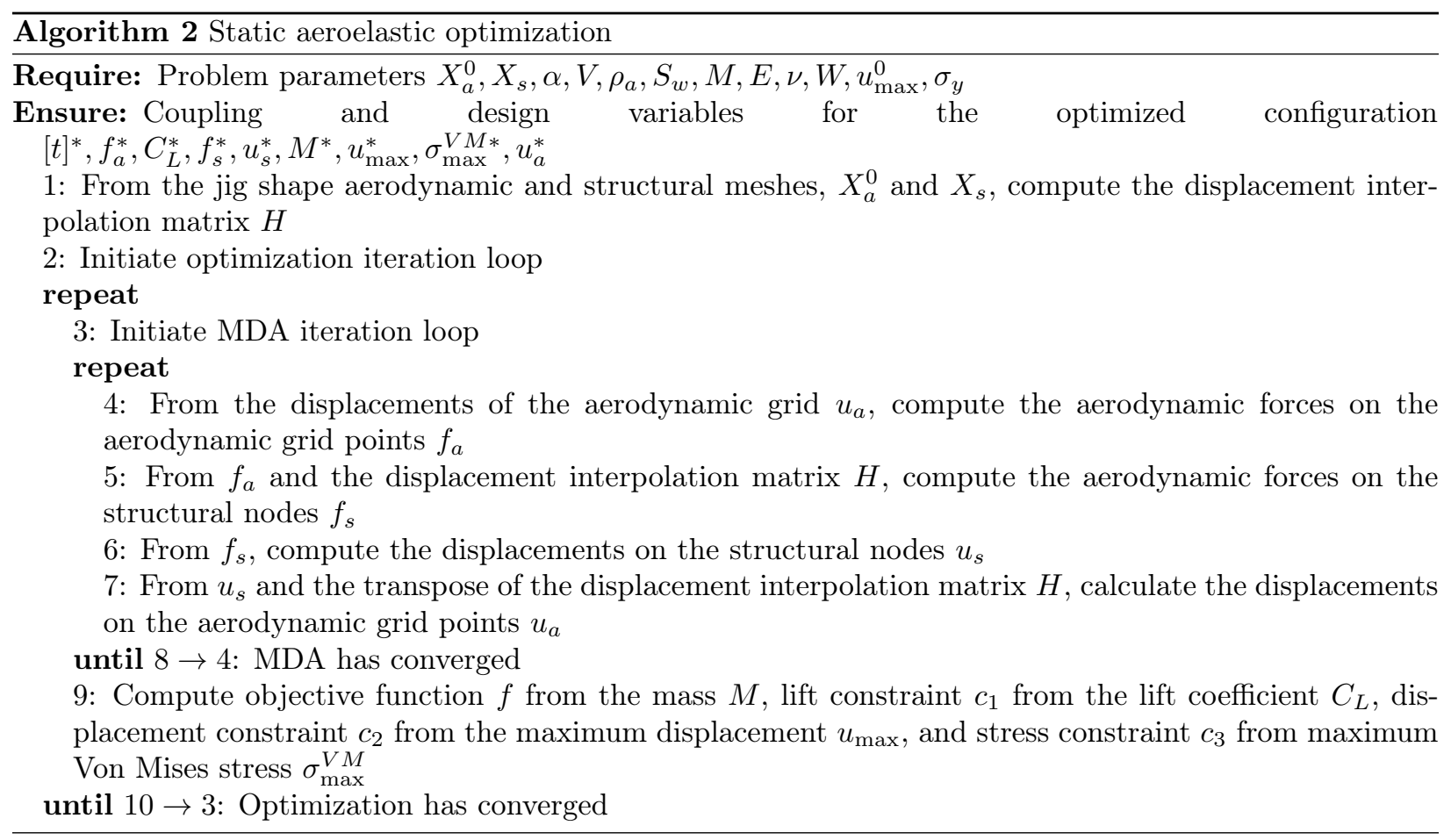




\section{B. Global Optimizer}

To solve the optimization problem described above, we use a sequential enrichment approach, typically the Efficient Global Optimization algorithm ${ }^{16}$ or Super EGO, ${ }^{17}$ an evolution of EGO to handle constraints. In some previous work, ${ }^{18}$ the proposed algorithm, called SEGOMOE, was successful to minimize the number of function evaluations. The idea of SEGOMOE is to use some adaptive mixture of kriging based models to tackle high dimension problems. The mixture of experts (MOE) ${ }^{19,20}$ are known to approximate complex functions with heterogeneous behaviour by combining local surrogate models in a global one. In order to consider high-dimensional functions to approximate (objective function and/or constraints), we used adapted local kriging-based models. ${ }^{21,22}$ The resulting coupling between SuperEGO and MOE, called SEGOMOE, is illustrated on Figure 11.

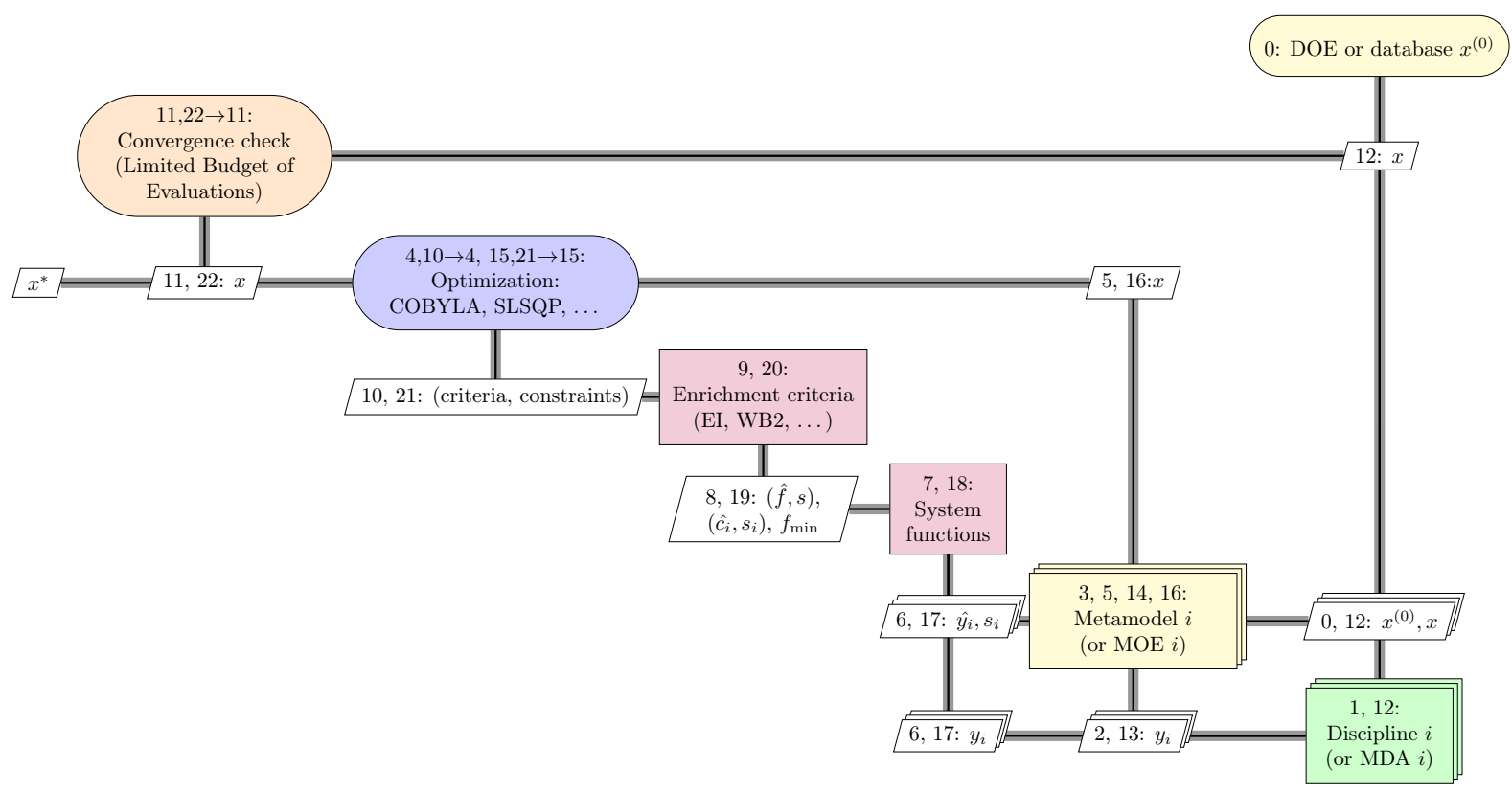

Figure 11. SEGOMOE algorithm.

The main steps are the following:

1. Construct the initial database (design of experiments) and build the associated MOE models relative to the objective function and the constraint functions.

2. Solve the optimization problem with enrichment criterion by maximizing the Expected Improvement subject to the constraints (bound constraints, yield stress, maximum displacement, ...) and propose the new enrichment point. In the original EGO algorithm, ${ }^{16}$ the mean and the variance of the objective function are required to compute the Expected Improvement criterion. In our case, they are given in exactly the same way by the kriging-based mixture of experts.

3. Compute the values of the objective function and the constraint functions at the new enrichment point and update the associated MOE models.

4. If the stopping criterion is not reached, return to Step 2.

SEGOMOE iterates until the stopping criterion is met. Due to the high computational cost of actual simulations, it is common to use the maximum number of function evaluations as the stopping criterion.

\section{Results}

The results of this optimization process are shown on figure 12, where the objective function and the violation of the constraints are plotted against the number of iterations. The first 75 iterations shown on figure 12 
correspond to the initial exploration of the design space (i.e., the creation of the design of experiments) to create the surrogate model of both the objective function and the constraints. Subsequent iterations correspond to the SEGOMOE algorithm itself.

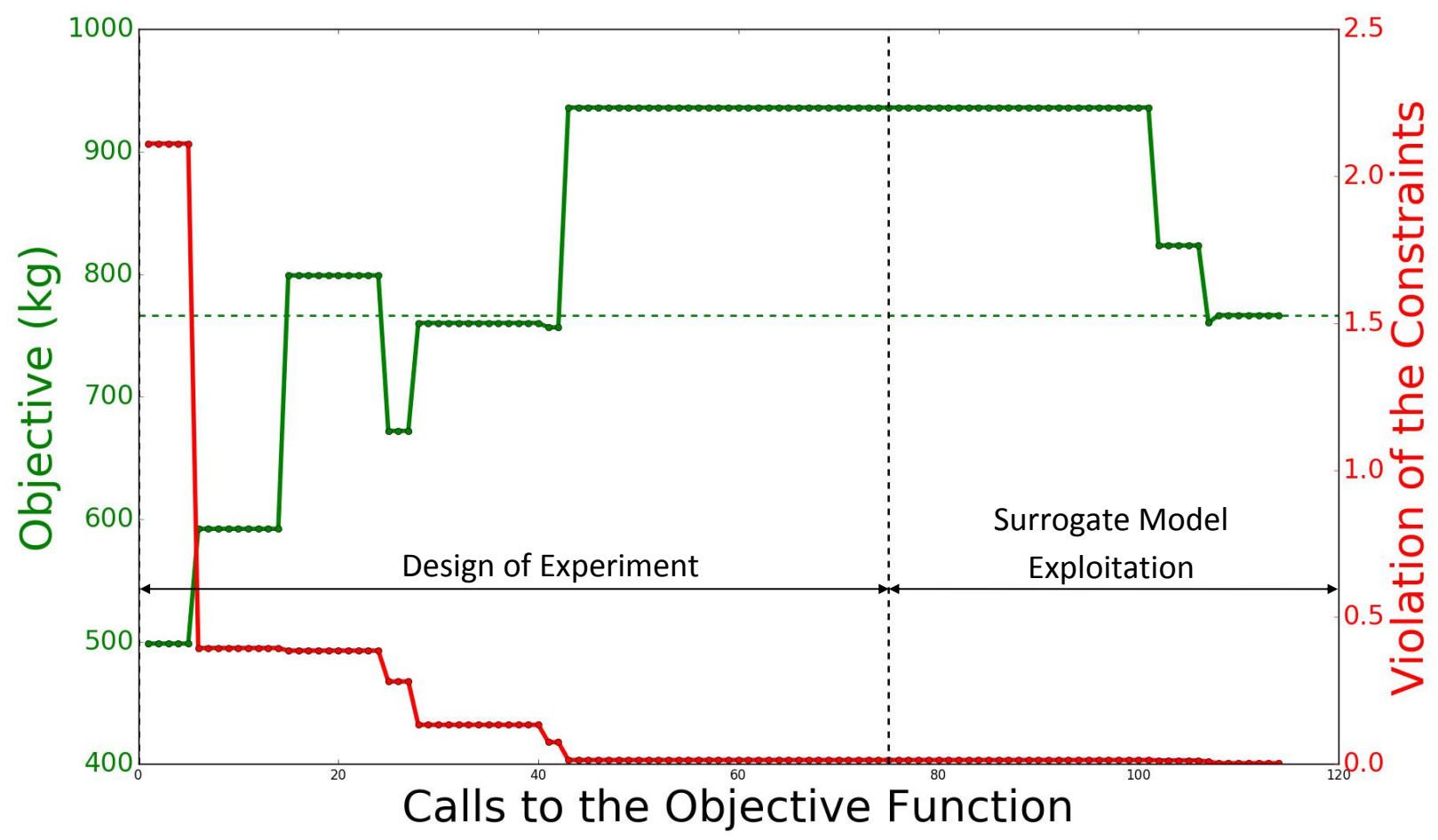

Figure 12. Static aeroelastic optimization results. All units are SI units.

We can see that the SEGOMOE optimizer converges towards a mass value of $769.32 \mathrm{~kg}$. Among the two inequality constraints (displacement and stress), only the displacement one was active at the optimum. This means the maximum prescribed displacement is reached before the von Mises stress is achieved on the wing structure. Though the allowable displacement is $30 \%$ of half the wingspan, the high flexibility of the wing makes the displacement constraint more critical than the stress one.

\section{Dynamic Aeroelastic Similarity}

In the work by Ricciardi et al., ${ }^{3}$ the dynamic aeroelastic equation of motion is nondimensionalized to establish the parameters that must be respected for the scaled model and the full scale aircraft to be aeroelastically similar (in the dynamic sense). By doing this dimensional analysis, a set of nondimensional parameters that are independent of the structural configuration must be respected. In addition, under the hypothesis of flow similarity, the nondimensional modal frequencies and shapes must be matched. In the general case where the whole structure (including the internal one) of the scaled model is not a perfectly scaled version of the full aircraft (e.g., if it uses a different spar and ribs distribution), the scaled modal frequencies and shapes cannot be obtained by simply scaling the material properties such as the modulus of elasticity, density or thicknesses. In that case, it is convenient to define a certain number of design variables of the scaled model (e.g., thicknesses or added masses) and to minimize the difference between the scaled modal frequencies and shapes. An aeroelastically scaled model, for example, has flutter speeds and frequencies that can be obtained by scaling those found on the full size aircraft.

Section A describes the traditional scaled modal optimization. Next, an optimization method to minimize the difference between the scaled modal frequencies and shapes is presented in Section B. Last, in Sections $\mathrm{C}$ and $\mathrm{D}$, a test case with a known reference solution for the modal optimization is presented. 


\section{A. Traditional Scaled Modal Optimization}

As explained above, the aeroelastic similarity, under the hypotheses by Ricciardi et al., ${ }^{3}$ can be achieved by minimizing the difference between the nondimensional modal frequencies and shapes. There may exist multiple optimization problem formulations to minimize this difference, but the objective function for the traditional scaled modal optimization is, as detailed by Ricciardi et al., ${ }^{3}$

$$
\sum_{m=1}^{N}\left\|\{\bar{\phi}\}_{t, m}-\{\bar{\phi}\}_{r, m}\right\|,
$$

where $\{\bar{\phi}\}_{t, m}$ is the $m^{t h}$ normalized mode shape of the target model, $\{\bar{\phi}\}_{r, m}$ is the $m^{t h}$ normalized mode shape of the scaled model, and $N$ is the number of eigenpairs. The problem is constrained so that the reduced frequencies match so

$$
\omega_{t, m} \frac{b_{t}}{V_{t}}=\omega_{r, m} \frac{b_{r}}{V_{r}}, \quad m=1,2, \cdots, N
$$

where the subscript $t$ indicates the target model, subscript $r$ indicates the scaled model, $b$ indicates the wingspan, and $V$ is the airspeed.

Concerning the order of the normal modes, which has an effect on how Eqns. (10) and (11) are evaluated, the majority of structural analysis codes sort the normal modes according to the increasing values of their corresponding frequencies. If modes are compared in that order, with no further modification, this can lead to discontinuities in the objective function and discontinuities on the derivatives of the constraints as defined by Eqns. (10) and (11). ${ }^{7}$ These discontinuities appear when the order of modes of a different nature (e.g., bending and torsion) is swapped when their corresponding frequencies intersect.

To avoid these drawbacks, modes can be compared by sorting them according to the nature of their shape. ${ }^{7}$ The idea behind this reordering of the modes is to find the mode shape of the current optimization iteration, for each of the mode shapes of the target model $\{\bar{\phi}\}_{t, m}$, whose shape is the closest to the target one. In that way, the values of the objective and constraints functions no longer depend on the order of the modes according to their eigenvalues. Instead, they are evaluated in agreement with the closeness between the target and current mode shapes.

To further illustrate this concept, let us imagine a case where we have a structure (e.g., a rectangular cross-sectional beam with a distributed added mass) with two bending modes in two different planes, and a structural design variable $x$ (e.g., the width of the beam). In that case, there is an intersection of the frequencies of the two modes, when the value of $x$ equals the height of the cross-section. This situation is illustrated in Figure 13, where we can see that the frequency function is not differentiable at the intersection if modes are sorted according to their eigenvalues. However, this is not the case when modes are ordered according to their shape.

Let us now consider the case of the functions of interest that depend on the values of the eigenvectors. If these are evaluated with the modes sorted according to their eigenvalues, these functions may exhibit discontinuities at the intersections of the eigenvalues. This happens as the components of the $i^{\text {th }}$ eigenvector change suddenly if another mode shape occupies the $i^{\text {th }}$ position after their corresponding eigenvalues have crossed, and thus their orders have swapped.

\section{B. Proposed Modal Optimization}

In this section, we propose an optimization formulation that avoids the drawbacks presented above. Modes can be tracked by using the Modal Assurance Criterion (MAC), which is a value that quantifies the similitude between two modes. ${ }^{7}$ The MAC between two mode shapes $\Phi_{a}$ and $\Phi_{b}$ is defined as

$$
\operatorname{MAC}\left(\Phi_{a}, \Phi_{b}\right)=\frac{\left\|\Phi_{a}^{T} \Phi_{b}\right\|}{\left(\Phi_{a}^{T} \Phi_{a}\right)\left(\Phi_{b}^{T} \Phi_{b}\right)},
$$

which is the normed scalar product between the two vectors. ${ }^{23}$ If the MAC value equals one, the two vectors represent the same mode, whereas if it is zero, they are orthogonal. If we define a matrix with the MAC values for all the scaled mode pairs between the full aircraft and the scaled model, we can associate each scaled model mode to the full aircraft mode whose MAC value is the closest to one. By reordering the scaled model modes in that way, we can smooth out the functions of interest mentioned above. Note that 

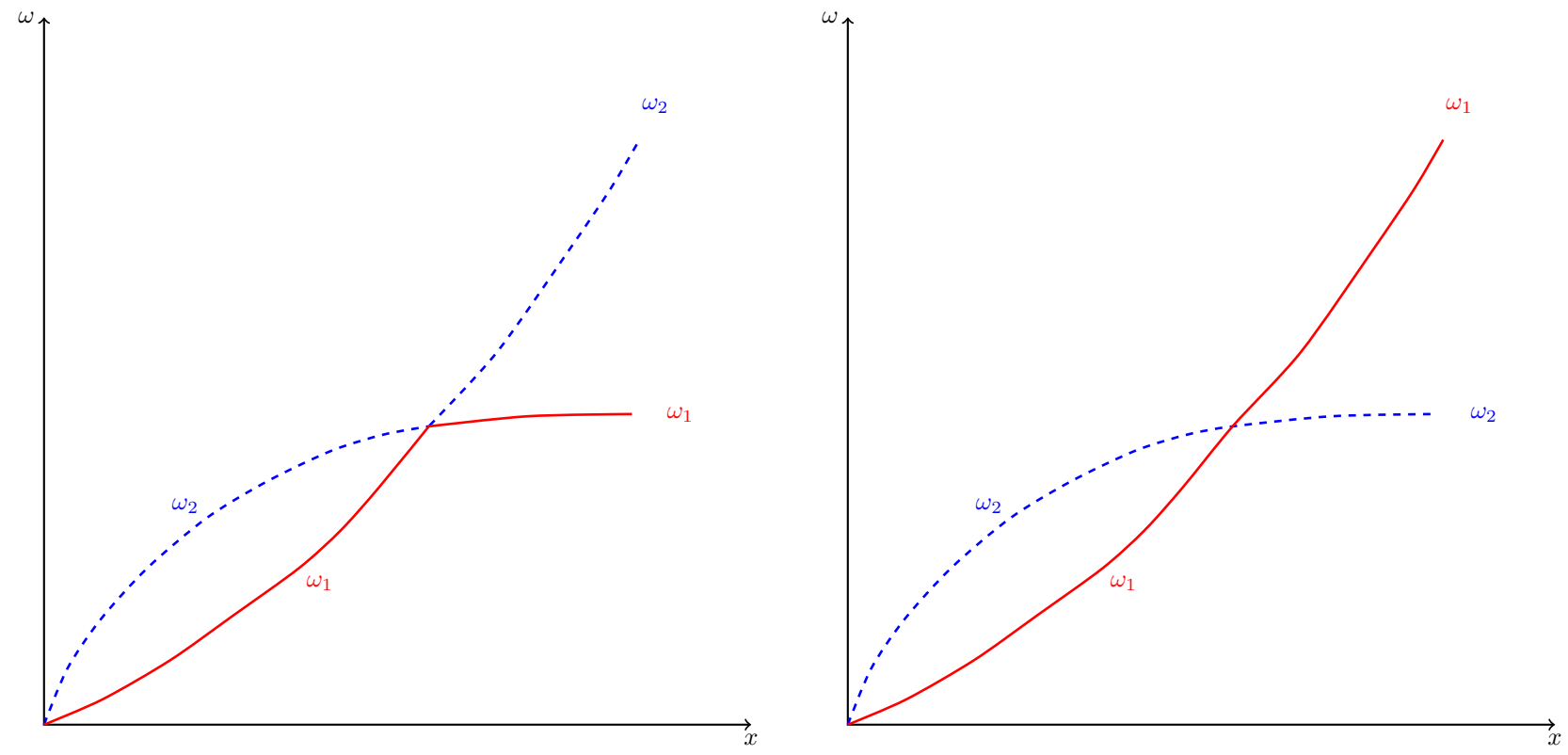

Figure 13. Beam frequencies sorted according to eigenvalues (left) and according to their mode shape (right).

this method assumes that for each reference mode, there exists only one scaled model mode for which the maximum MAC value is the one that corresponds to this reference mode.

Apart from the reordering of the modes, the MAC can also be used to avoid other problems that can emerge from the use of functions of the form of equation (10). This can be the case for two slightly different vectors that qualitatively represent the same mode, but whose displacement coordinates have opposite signs due to how the normalization of the vectors is performed (for example, normalized so the maximum displacement component equals to 1 ). In that case, the objective function represented by equation (10) would be penalized, whereas the two vectors represent nearly the same modal shape. This phenomenon is illustrated in Figure 14 and can appear, for example, on antisymmetric or torsion modes.

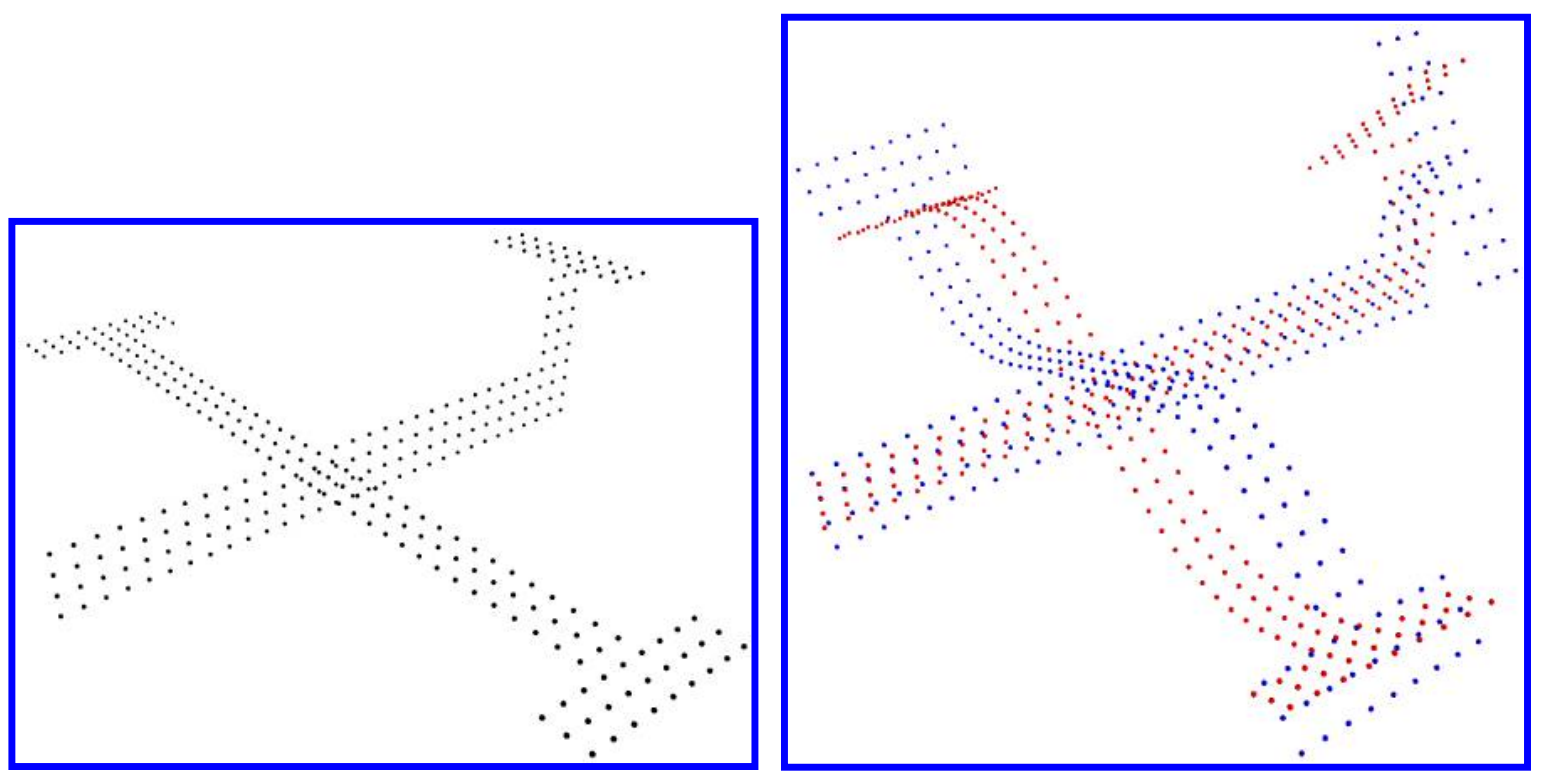

Figure 14. Non-deformed GARTEUR model (left). Two similar antisymmetric modes (right). 
In the ideal case where there is no difference between the target modes and the optimized modes (after they have been sorted according to the MAC matrix), their corresponding newly ordered MAC matrix is the identity matrix. Since the MAC value is between 0 and 1 , the number of mode pairs considered $N$ constitutes an upper bound on the trace of the MAC matrix. Therefore, we can use the trace of the MAC matrix as the objective function to maximize in order to avoid the problems mentioned on the previous paragraph.

\section{Problem Formulation}

A first test case for the modal optimization process, described in Table 4, consists in finding the plate thicknesses and concentrated masses of the GARTEUR SM-AG19 model in order to match the first 10 modes and frequencies of the reference configuration. This model, presented by Link and Friswell, ${ }^{8}$ is shown in Figure 15. The optimization variables are the thicknesses of the fuselage and wing plates, and the point mass values at the wingtips and at the empennage, resulting in a total of 5 design variables.

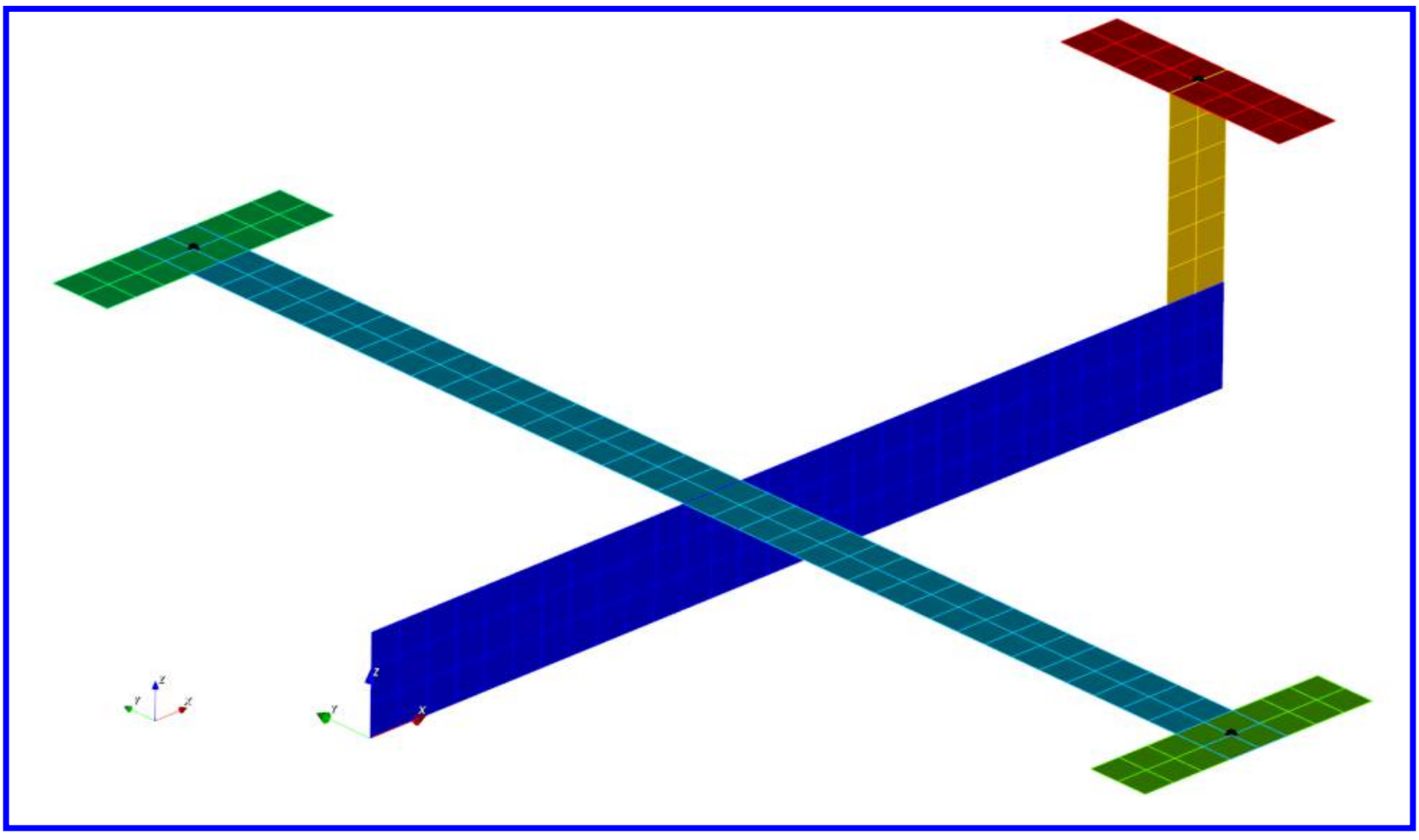

Figure 15. Finite element model of the GARTEUR testbed. ${ }^{8}$ Point masses are represented as black dots.

Objective Function

Dimension Bounds

\begin{tabular}{lccl}
\hline $\begin{array}{l}\text { MAC matrix trace maximization } \\
\text { Design Variables }\end{array}$ & $\max (\operatorname{trace}(\mathrm{MAC}))$ & $\mathbb{R}$ & \\
\hline Plate thicknesses vector & {$[t]$} & $\mathbb{R}^{2}$ & {$[1,60] \mathrm{mm}$} \\
Concentrated masses vector & {$[m]$} & $\mathbb{R}^{3}$ & {$[0.01,0.6] \mathrm{kg}$} \\
Constraints & & & \\
\hline Reduced frequency matching & $\left\|\boldsymbol{\omega}_{t} \frac{b_{t}}{V_{t}}-\boldsymbol{\omega}_{r} \frac{b_{r}}{V_{r}}\right\|=0$ & $\mathbb{R}$ & \\
Mass matching & $M-M_{0}=0$ & $\mathbb{R}$ &
\end{tabular}

Table 4. Dynamic aerostructural optimization problem.

Figure 16 shows the XDSM diagram corresponding to the presented modal optimization process. 


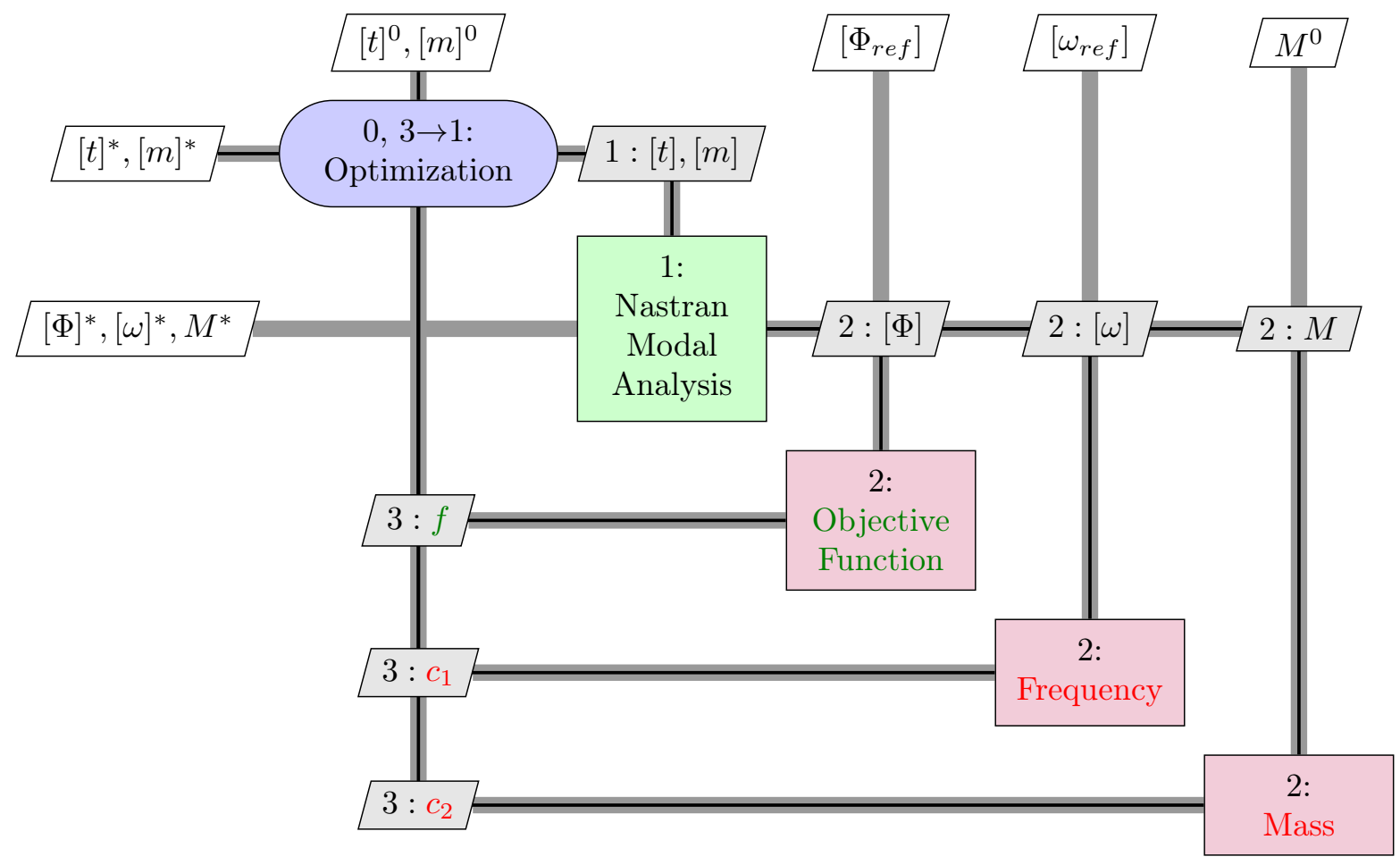

Figure 16. XDSM representation of the implemented modal optimization.

Algorithm 3 describes the sequence of operations performed to carry out the multidisciplinary analysis and optimization shown on figure 16.

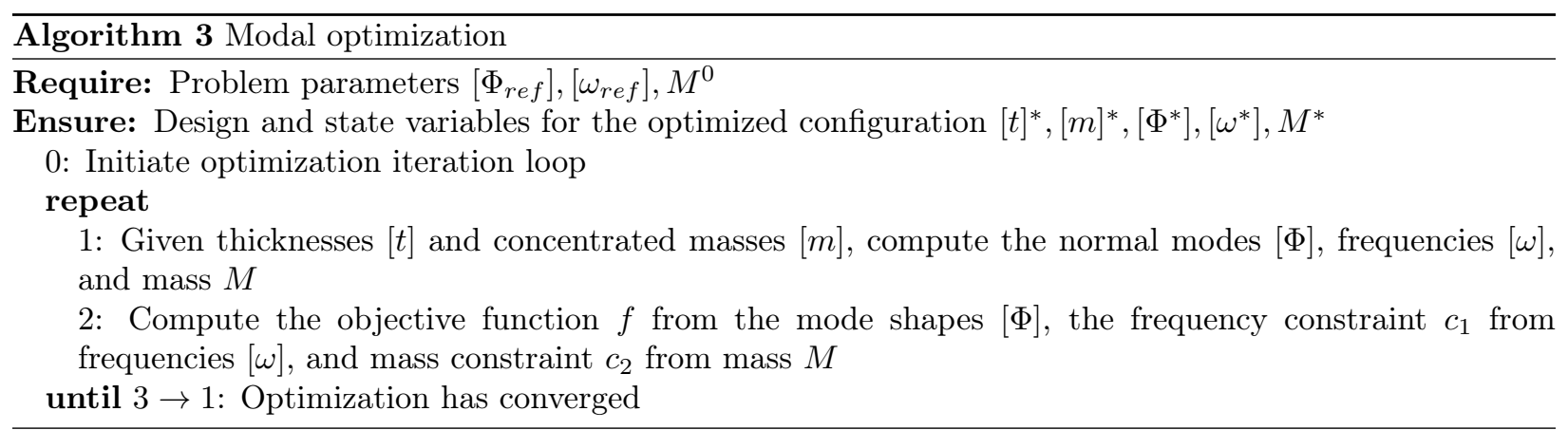

\section{Results}

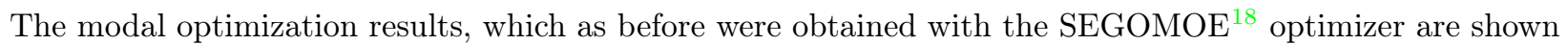
on Figure 17, where the objective function and the violation of constraints are plotted against the number of iterations. The first 100 iterations correspond to the design of experiments used to build the surrogate model. The subsequent iterations correspond to the exploitation of the surrogate model by the SEGOMOE algorithm in order to minimize the objective function. 


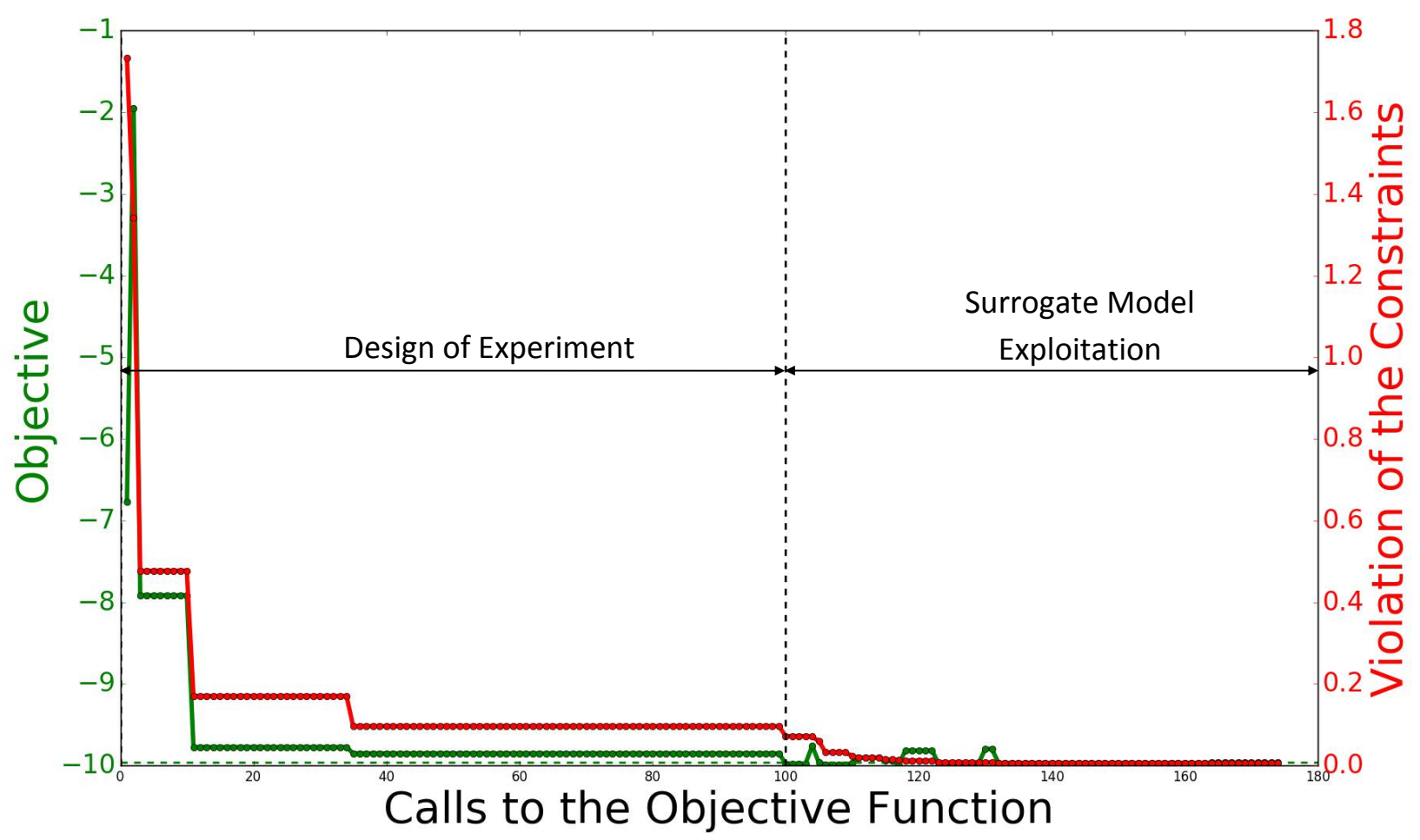

Figure 17. Modal optimization results. All units are SI units.

Note that, as formulated in table 4, this was a maximization problem. However, since it is implemented as a minimization problem on the SEGOMOE optimizer, the sign of the objective function has been changed. As we are considering the first 10 vibration modes, the trace of the MAC matrix is bounded by a value of 10, as explained in Section B. We can see in Figure 17 that the objective function tends to the bound value of -10 while the frequency and mass constraints tend to zero, as expected.

\section{Conclusion}

The use of aeroelastically scaled models for the study of new aircraft configurations presents itself as a means of testing novel plane concepts, without the risk of developing and building a full size aircraft. When flow similarity (e.g., same Mach number) does not exist between the model and the aircraft, the same scaled deformed shape cannot be obtained by simply scaling the stiffness and preserving of the outer mold line. This is the reason for the development of the aeroelastic coupling presented on the first part of this work. In the future, this aeroelastic coupling will allow for the optimization of the structural properties in order to obtain a scaled deformed shape in the case where there exists no flow similarity.

We validated this MDA against a benchmark case of a HALE wing, giving a relative error of $0.37 \%$ in the wingtip deflection. Once the implementation of this MDA process was validated, we used it in an optimization problem where the objective function was of the classical type: mass minimization. In the future, an objective function related to the wing displacements for aeroelastic similarity purposes will be used.

We also considered dynamic aeroelastic similarity, and as a first approach, we used the classical method where the aerodynamic similarity is assumed through the use of a scaled version of the outer mold line. This assumption leads to a structural modal optimization problem. We presented the traditional scaled modal optimization and introduced the use of the Modal Assurance Criterion both for reordering the normal modes according to their shape and for the definition of the objective function. This method should avoid the discontinuities on the objective function and on the derivatives of the constraints. Last, we tested the proposed optimization approach on a case based on the GARTEUR SM-AG19 model, whose solution 
corresponds to the known reference configuration.

Future developments will include the formulation and implementation of static and aeroelastic similarities on a single MDO problem. The classical hypotheses and assumptions of the classical aeroelastic dynamic similarity should be reconsidered, as we did with the static case for the flow similarity. Also, new design variables other than thicknesses and masses should be considered to modify the stiffness of the wing. An example of this is the use of piezoelectric patches to modify the local rigidity.

\section{References}

${ }^{1}$ Richards, J., Suleman, A., Aarons, T., and Canfield, R., "Multidisciplinary Design for Flight Test of a Scaled Joined Wing SensorCraft," AIAA MAO Conference, 2010.

${ }^{2}$ Ricciardi, A. P., Canfield, R. A., Patil, M. J., and Lindsley, N., "Nonlinear Aeroelastic Scaled-Model Design," Journal of Aircraft, Vol. 53, No. 1, Jan. 2016, pp. 20-32.

${ }^{3}$ Ricciardi, A., Canfield, R. A., Patil, M., Eger, C. A., and Lindsley, N. J., "Nonlinear Aeroelastic Scaled Model Optimization Using Equivalent Static Loads," American Institute of Aeronautics and Astronautics, April 2013.

${ }^{4}$ Lambe, A. B. and Martins, J. R., "Extensions to the design structure matrix for the description of multidisciplinary design, analysis, and optimization processes," Structural and Multidisciplinary Optimization, Vol. 46, No. 2, 2012, pp. $273-284$.

${ }^{5}$ Gray, J., Hearn, T., Moore, K., Hwang, J. T., Martins, J. R. R. A., and Ning, A., "Automatic Evaluation of Multidisciplinary Derivatives Using a Graph-Based Problem Formulation in OpenMDAO," Proceedings of the 15th AIAA/ISSMO Multidisciplinary Analysis and Optimization Conference, Atlanta, GA, June 2014.

${ }^{6}$ Patil, M. and Hodges, D., "On the importance of aerodynamic and structural geometrical nonlinearities in aeroelastic behavior of high-aspect-ratio wings," Journal of Fluids and Structures, Vol. 19, No. 7, Aug. 2004, pp. 905-915.

${ }^{7} \mathrm{Kim}, \mathrm{T}$. and Kim, Y., "Mac-based mode-tracking in structural topology optimization," Computers and Structures, Vol. 74, 2000, pp. 375-383.

${ }^{8}$ Link, M. and Friswell, M., "WORKING GROUP 1: GENERATION OF VALIDATED STRUCTURAL DYNAMIC MODELS-RESULTS OF A BENCHMARK STUDY UTILISING THE GARTEUR SM-AG19 TEST-BED," Mechanical Systems and Signal Processing, Vol. 17, No. 1, Jan. 2003, pp. 9-20.

${ }^{9}$ The NASTRAN user's manual: level 16.0, Scientific and Technical Information Office, National Aeronautics and Space Administration, 1976.

${ }^{10}$ A5021 User's Manual-PAN AIR Technology Program for Solving Problems of Potential Flow about Arbitrary Configurations, Boeing, 1992.

${ }^{11}$ Rendall, T. C. S. and Allen, C. B., "Unified fluid-structure interpolation and mesh motion using radial basis functions," International Journal for Numerical Methods in Engineering, Vol. 74, No. 10, June 2008, pp. 1519-1559.

12 Jakobsson, S. and Amoignon, O., "Mesh deformation using radial basis functions for gradient-based aerodynamic shape optimization," Computers \& Fluids, Vol. 36, No. 6, July 2007, pp. 1119-1136.

${ }^{13}$ Lombardi, M., Parolini, N., and Quarteroni, A., "Radial basis functions for inter-grid interpolation and mesh motion in FSI problems," Computer Methods in Applied Mechanics and Engineering, Vol. 256, April 2013, pp. 117-131.

${ }^{14}$ Hallissy, B. P. and Cesnik, C. E., "High-fidelity aeroelastic analysis of very flexible aircraft," Proceedings of the 52th AIAA/ASME/ASCE/AHS/ASC Structures, Structural Dynamics, and Materials Conference, 2011, pp. 2011-1914.

$\checkmark{ }^{15}$ Martins, J. R. R. A. and Lambe, A. B., "Multidisciplinary Design Optimization: A Survey of Architectures," AIAA Journal, Vol. 51, No. 9, September 2013, pp. 2049-2075.

${ }^{16}$ Jones, D. R., Schonlau, M., and Welch, W. J., "Efficient global optimization of expensive black-box functions," Journal of Global optimization, Vol. 13, No. 4, 1998, pp. 455-492.

${ }^{17}$ Sasena, M. J., Flexibility and efficiency enhancements for constrained global design optimization with kriging approximations, Ph.D. thesis, General Motors, 2002.

${ }^{18}$ Bartoli, N., Kurek, I., Lafage, R., Lefebvre, T., Priem, R., Bouhlel, M.-A., Morller, J., Stilz, V., and Regis, R., "Improvement of efficient global optimization with mixture of experts: methodology developments and preliminary results in aircraft wing design," 17th AIAA/ISSMO Multidisciplinary Analysis and Optimization Conference, Washington D.C., USA, June 2016 2016.

${ }^{19}$ Bettebghor, D., Bartoli, N., Grihon, S., Morlier, J., and Samuelides, M., "Surrogate modeling approximation using a mixture of experts based on EM joint estimation," Structural and Multidisciplinary Optimization, Vol. 43, No. 2, 2011, pp. 243-259, 10.1007/s00158-010-0554-2.

${ }^{20}$ Liem, R. P. and Martins, J. R. R. A., "Surrogate Models and Mixtures of Experts in Aerodynamic Performance Prediction for Mission Analysis," 15th AIAA/ISSMO Multidisciplinary Analysis and Optimization Conference, No. AIAA-2014-2301., Atlanta, GA, June 20142014.

${ }^{21}$ Bouhlel, M. A., Bartoli, N., Otsmane, A., and Morlier, J., "Improving kriging surrogates of high-dimensional design models by Partial Least Squares dimension reduction," Structural and Multidisciplinary Optimization, Vol. 53, No. 5, 2016, pp. 935-952.

${ }^{22}$ Bouhlel, M. A., Bartoli, N., Otsmane, A., and Morlier, J., "An Improved Approach for Estimating the Hyperparameters of the Kriging Model for High-Dimensional Problems through the Partial Least Squares Method," Mathematical Problems in Engineering, Vol. 2016, 2016.

${ }^{23}$ Girard, A. and Roy, N., Structural dynamics in industry, Wiley, 2008. 